\title{
Convergence of Probability Measures and Markov Decision Models with Incomplete Information
}

\author{
July 4, 2014 \\ Eugene A. Feinberg 1, Pavlo O. Kasyanov2, and Michael Z. Zgurovsky 3 \\ This article is dedicated to 80th birthday of Academician Albert Nikolaevich Shiryaev
}

\begin{abstract}
This paper deals with three major types of convergence of probability measures on metric spaces: weak convergence, setwise converges, and convergence in the total variation. First, it describes and compares necessary and sufficient conditions for these types of convergence, some of which are well-known, in terms of convergence of probabilities of open and closed sets and, for the probabilities on the real line, in terms of convergence of distribution functions. Second, it provides criteria for weak and setwise convergence of probability measures and continuity of stochastic kernels in terms of convergence of probabilities defined on the base of the topology generated by the metric. Third, it provides applications to control of Partially Observable Markov Decision Processes and, in particular, to Markov Decision Models with incomplete information.
\end{abstract}

\section{Introduction}

This paper deals with convergence of probability measures and relevant applications to control of stochastic systems with incomplete state observations. Convergence of probability measures and control of stochastic systems under incomplete information are among the areas to which Albert Nikolayevich Shiryaev has made fundamental contributions. In particular, convergence of probability measures and limit theorems for stochastic processes were studied in his joint papers with his distinguished students Yuri Mikhailovich Kabanov and Robert Shevilevich Liptser (e.g., [21]) and in his monograph with Jean Jacod [20]. Control of stochastic processes with incomplete information was the major topic of his two influential papers [28, 29], and this topic is related to his monograph with Liptser [22] on statistics of stochastic processes.

\footnotetext{
${ }^{1}$ Department of Applied Mathematics and Statistics, Stony Brook University, Stony Brook, NY 11794-3600, USA, eugene.feinberg@sunysb.edu

${ }^{2}$ Institute for Applied System Analysis, National Technical University of Ukraine "Kyiv Polytechnic Institute", Peremogy ave., 37, build, 35, 03056, Kyiv, Ukraine, kasyanov@i.ua.

${ }^{3}$ National Technical University of Ukraine "Kyiv Polytechnic Institute", Peremogy ave., 37, build, 1, 03056, Kyiv, Ukraine, zgurovsm@hotmail.com
} 
In Section 2 of this paper we describe three major types of convergence of probability measures defined on metric spaces: weak convergence, setwise convergence, and convergence in the total variation. In addition to the definitions, we provide two groups of mostly known results: characterizations of these types of convergence via convergence of probability measures of open and closed sets, and, for probabilities on a real line, via convergence of distribution functions. In section 3 we describe criteria for weak and setwise convergences in terms of convergence of probabilities of the elements of a countable base of the topology. Section 4 deals with continuity of transition probabilities. In particular, Theorem 4.4 describes sufficient conditions for a probability measure, defined on a product of two spaces and depending on a parameter, to have a transition probability satisfying certain continuity properties. This result can be interpreted as a sufficient condition for continuity in Bayes's formula. Section 5 describes recent results on optimization of Partially Observable Markov Decision Processes (POMDPs) from Feinberg et al. [15] as well as new results. Section 6 describes an application of the results from Sections 4 and 5 to a particular class of POMDPs, that we call Markov Decision Models with Incomplete Information (MDMIIs). The difference between a POMDP and an MDMII is that for a POMDP the states of the system and observations are related via a stochastic kernel, called an observation stochastic kernel, while for an MDMII the state of the system is a vector, consisting of $(m+n)$ coordinates, of which $m$ coordinates are observable and $n$ coordinates are not observable. MDMIIs were studied mainly in early publications including in Aoki [1], Dynkin [9], Shiryaev [29], Hinderer [19], Savarigi and Yoshikava [27], Rhenius [24], Rieder [25], Yushkevich [34], Dynkin and Yushkevich [10], and Bäuerle and Rieder [3], while POMDPs were studied by Bertsekas and Shreve [5], Hernández-Lerma [17], and in many later publications.

Feinberg et al. [15] described sufficient conditions for the existence of optimal policies, validity of optimality equations, and convergence of value iterations to optimal values for POMDPs with standard Borel state, action, and observation spaces and for MDMIIs with standard Borel state and action spaces; see also conference and seminar proceedings [14, 16]. In both cases, the goal is either to minimize the expected total costs, with the one-step cost function being nonnegative, or to minimize the expected total discounted cost, with the one-step cost function being bounded below. For POMDPs these sufficient conditions are: $K$-inf-compactness of the cost function, weak continuity of the transition stochastic kernel, and continuity in the total variation of the observation stochastic kernel. These results are described in Section 5 as well as sufficient conditions for weak continuity of transition probabilities for a COMDP from Feinberg et al. [15] in terms of the transition function $H$ in the filtering equation (5.4). In this paper we introduce sufficient conditions in terms of joint distributions of posteriory distributions and observations; see Theorem 5.5 The notion of $K$-inf-compactness of a function defined on a graph of a set-valued map was introduced in Feinberg et al. [13].

Though an MDMII is a particular case of an POMDP, there is no observation stochastic kernel in the definition of an MDMII. However, the observation stochastic kernel can be defined for an MDMII in a natural way, and this definition transforms an MDMII into a POMDP, but in this POMDP the defined observation stochastic kernel is not continuous in the total variation. Feinberg et al. [15] described additional equicontinuity conditions on the stochastic kernels of MDMIIs, under which optimal policies exist, optimality equations hold, and value iterations converge to optimal values. By using results from Sections 4 and 5, in Section 6 we strengthen the results from Feinberg et al. [15] on MDMIIs by providing weaker 
assumptions on transition probabilities than the assumptions introduced in Feinberg et al. [15].

\section{Three types of convergence of probability measures}

Let $\mathbb{S}$ be a metric space and $\mathcal{B}(\mathbb{S})$ be its Borel $\sigma$-field, that is, the $\sigma$-field generated by all open subsets of the metric space $\mathbb{S}$. For $S \in \mathcal{B}(\mathbb{S})$ denote by $\mathcal{B}(S)$ the $\sigma$-field whose elements are intersections of $S$ with elements of $\mathcal{B}(\mathbb{S})$. Observe that $S$ is a metric space with the same metric as on $\mathbb{S}$, and $\mathcal{B}(S)$ is its Borel $\sigma$-field. For a metric space $\mathbb{S}$, denote by $\mathbb{P}(\mathbb{S})$ the set of probability measures on $(\mathbb{S}, \mathcal{B}(\mathbb{S}))$. A sequence of probability measures $\left\{P_{n}\right\}_{n=1,2, \ldots}$ from $\mathbb{P}(\mathbb{S})$ converges weakly (setwise) to $P \in \mathbb{P}(\mathbb{S})$ if for any bounded continuous (bounded Borel-measurable) function $f$ on $\mathbb{S}$

$$
\int_{\mathbb{S}} f(s) P_{n}(d s) \rightarrow \int_{\mathbb{S}} f(s) P(d s) \quad \text { as } \quad n \rightarrow \infty .
$$

We write $P_{n} \stackrel{w}{\longrightarrow} P\left(P_{n} \stackrel{s}{\longrightarrow} P\right)$ if the sequence $\left\{P_{n}\right\}_{n=1,2, \ldots}$ from $\mathbb{P}(\mathbb{S})$ converges weakly (setwise) to $P \in$ $\mathbb{P}(\mathbb{S})$. The definition of Lebesgue-Stiltjes integrals implies that $P_{n} \stackrel{s}{\longrightarrow} P$ if and only if $P_{n}(E) \rightarrow P(E)$ for each $E \in \mathcal{B}(\mathbb{S})$ as $n \rightarrow \infty$. The following two theorems are well-known.

Theorem 2.1. (Shiryaev [30, Theorem 1, p. 311]). The following statements are equivalent:

(i) $P_{n} \stackrel{w}{\longrightarrow} P$;

(ii) $\liminf _{n \rightarrow \infty} P_{n}(\mathcal{O}) \geq P(\mathcal{O})$ for each open subset $\mathcal{O} \subseteq \mathbb{S}$;

(iii) $\limsup _{n \rightarrow \infty} P_{n}(C) \leq P(C)$ for each closed subset $C \subseteq \mathbb{S}$.

Let $\mathbb{R}^{1}$ be a real line with the Euclidean metric. For a $P, P_{n} \in \mathbb{P}\left(\mathbb{R}^{1}\right)$ define the distribution functions $F(x)=P\{(-\infty, x]\}$ and $F_{n}(x)=P_{n}\{(-\infty, x]\}, x \in \mathbb{R}^{1}$.

Theorem 2.2. (Shiryaev [30, Theorem 2, p. 314]). For $\mathbb{S}=\mathbb{R}^{1}$ the following statements are equivalent:

(i) $P_{n} \stackrel{w}{\longrightarrow} P$;

(ii) $F_{n}(x) \rightarrow F(x)$ for all points $x \in \mathbb{R}^{1}$ of continuity of the distribution function $F$.

The following theorem provides for setwise convergence the results in the same spirit as Theorem 2.1 states for weak convergence.

Theorem 2.3. The following statements are equivalent:

(i) $P_{n} \stackrel{s}{\longrightarrow} P$;

(ii) $\lim _{n \rightarrow \infty} P_{n}(\mathcal{O})=P(\mathcal{O})$ for each open subset $\mathcal{O} \subseteq \mathbb{S}$;

(iii) $\lim _{n \rightarrow \infty} P_{n}(C)=P(C)$ for each closed subset $C \subseteq \mathbb{S}$.

Proof. If $A$ is open (closed) then its complement $A^{c}$ is closed (open), and $Q\left(A^{c}\right)=1-Q(A)$ for each $Q \in \mathbb{P}(\mathbb{S})$. Thus statements (ii) and (iii) are equivalent. We prove the equivalence of (i) and (iii). Obviously, (i) implies (iii). According to Billingsley [6, Theorem 1.1] or Bogachev [7, Theorem 7.1.7], any probability measure $P$ on a metric space $\mathbb{S}$ is regular, that is, for each $B \in \mathcal{B}(\mathbb{S})$ and for each $\varepsilon>0$ there exist a closed subset $C \subseteq \mathbb{S}$ and an open subset $\mathcal{O} \subseteq \mathbb{S}$ such that $C \subseteq B \subseteq \mathcal{O}$ and $P(\mathcal{O} \backslash C)<\varepsilon$. Fix arbitrary $B \in \mathcal{B}(\mathbb{S})$ and $\varepsilon>0$. Since $P_{n}(\mathcal{O}) \rightarrow P(\mathcal{O})$ and $P_{n}(C) \rightarrow P(C)$, there exists $N=1,2, \ldots$, such that 
$\left|P_{n}(\mathcal{O})-P(\mathcal{O})\right|<\varepsilon$ and $\left|P_{n}(C)-P(C)\right|<\varepsilon$ for any $n=N, N+1, \ldots$ Therefore, $P_{n}(B)-P(B) \leq$ $P_{n}(\mathcal{O})-P(B)<\varepsilon+P(\mathcal{O} \backslash C)<2 \varepsilon$, and $P(B)-P_{n}(B) \leq P(B)-P_{n}(C)<\varepsilon+P(\mathcal{O} \backslash C)<2 \varepsilon$, for each $n=N, N+1, \ldots$ Since $\varepsilon>0$ is arbitrary, the sequence $\left\{P_{n}(B)\right\}_{n=1,2, \ldots} \subset[0,1]$ converges to $P(B)$ for any $B \in \mathcal{B}(\mathbb{S})$, that is, the sequence of probability measures $\left\{P_{n}\right\}_{n=1,2, \ldots}$ converges setwise to $P \in \mathbb{P}(\mathbb{S})$.

According to Bogachev [7, Theorem 8.10.56], which is Pflanzagl's generalization of the FichtengolzDieudonné-Grothendiek theorem, the statement of Theorem 2.3 holds for Radon measures. In view of Bogachev [7, Theorem 7.1.7], if $\mathbb{S}$ is complete and separable, then any probability measure on $(\mathbb{S}, \mathcal{B}(\mathbb{S}))$ is Radon. However, Theorem 2.3 does not assume that $\mathbb{S}$ is either separable or complete.

If $P_{n} \stackrel{s}{\longrightarrow} P$, where $P, P_{n} \in \mathbb{P}\left(\mathbb{R}^{1}\right)$ for all $n=1,2, \ldots$, then $F_{n}(x) \rightarrow F(x)$ and $F_{n}(x-) \rightarrow F(x-)$ for all $x \in \mathbb{R}^{1}$. This is true because $F_{n}(x)=P_{n}((-\infty, x]) \rightarrow P((-\infty, x])=F(x)$ and $F_{n}(x-)=$ $P_{n}((-\infty, x)) \rightarrow P((-\infty, x))=F(x-)$ as $n \rightarrow \infty$. However, as the following example shows, the convergences $F_{n}(x) \rightarrow F(x)$ and $F_{n}(x-) \rightarrow F(x-)$ for all $x \in \mathbb{R}^{1}$ do not imply $P_{n} \stackrel{s}{\longrightarrow} P$.

Example 2.4. (Convergences $F_{n}(x) \rightarrow F(x)$ and $F_{n}(x-) \rightarrow F(x-) \forall x \in \mathbb{R}^{1}$ do not imply $P_{n} \stackrel{s}{\longrightarrow} P$ ). Let $F_{0}(x):= \begin{cases}0, & x<0 ; \\ x, & 0 \leq x \leq 1 ; \quad F_{n+1}(x):=\left\{\begin{array}{ll}\frac{1}{2} F_{n}(3 x), & x<\frac{1}{3} ; \\ \frac{1}{2}, & \frac{1}{3} \leq x \leq \frac{2}{3} ; \quad F(x):=\left\{\begin{array}{ll}0, & x<0 ; \\ 1, & x>1 ;\end{array} \quad\{(x), \quad 0 \leq x \leq 1 ;\right. \\ \frac{1}{2} F_{n}(3 x-2), & x>\frac{2}{3} ;\end{array} \quad x>1 ;\right.\end{cases}$ where $C(x)$ is the Cantor function and $n=0,1, \ldots$. Note that $F(x)$ and $F_{n}(x), n=0,1, \ldots$, are continuous functions and

$$
\max _{x \in \mathbb{R}^{1}}\left|F(x)-F_{n}(x)\right| \leq 2^{1-n} \max _{x \in \mathbb{R}^{1}}\left|F_{1}(x)-F_{0}(x)\right|, \quad n=1,2, \ldots .
$$

Therefore, $F_{n}(x-)=F_{n}(x) \rightarrow F(x)=F(x-)$ for each $x \in \mathbb{R}^{1}$.

Denote by $C \subset[0,1]$ the Cantor set. Since the Lebesgue measure of the Cantor set $C$ equals zero and each distribution function $F_{n}$ has a bounded density, $P_{n}(C)=0$ for each $n=1,2, \ldots$ Note that $P(C)=1$ because $P([0,1])=F(1)-F(0)=1$ and $P([0,1] \backslash C)=0$ since $[0,1] \backslash C$ is a union of disjoint open interval each of zero $P$-measure. Thus, the sequence of probability measures $\left\{P_{n}\right\}_{n=1,2, \ldots}$ does not converges setwise to the probability measure $P$.

The third major type of convergence of probability measures, convergence in the total variation, can be defined via a metric $\rho_{t v}$ on $\mathbb{P}(\mathbb{S})$ called the distance in the total variation. For $P, Q \in \mathbb{P}(\mathbb{S})$, define

$$
\rho_{t v}(P, Q):=\sup \left\{\left|\int_{\mathbb{S}} f(s) P(d s)-\int_{\mathbb{S}} f(s) Q(d s)\right|: f: \mathbb{S} \rightarrow[-1,1] \text { is Borel-measurable }\right\} .
$$

A sequence of probability measures $\left\{P_{n}\right\}_{n=1,2, \ldots}$ from $\mathbb{P}(\mathbb{S})$ converges in the total variation to $P \in \mathbb{P}(\mathbb{S})$ if $\lim _{n \rightarrow \infty} \rho_{t v}\left(P_{n}, P\right)=0$.

In view of the Hahn decomposition, there exists $E \in \mathcal{B}(\mathbb{S})$ such that $(P-Q)(B) \geq 0$ for each $B \in \mathcal{B}(E)$ and $(P-Q)(B) \leq 0$ for each $B \in \mathcal{B}\left(E^{c}\right)$. According to Shiryaev [30, p. 360],

$$
\rho_{t v}(P, Q)=P(E)-Q(E)+Q\left(E^{c}\right)-P\left(E^{c}\right)=2 \sup \{|P(B)-Q(B)|: B \in \mathcal{B}(\mathbb{S})\} .
$$


This implies that the supremum in (2.1) is achieved at the function $f(s)=\mathbf{I}\{s \in E\}-\mathbf{I}\left\{s \in E^{c}\right\}$, and

$$
\rho_{t v}(P, Q)=\sup \left\{\int_{\mathbb{S}} f(s) P(d s)-\int_{\mathbb{S}} f(s) Q(d s): f: \mathbb{S} \rightarrow\{-1,1\} \text { is Borel-measurable }\right\} .
$$

Since $(P-Q)(\mathbb{S})=0,2.2)$ also implies

$$
\rho_{t v}(P, Q)=2 P(E)-2 Q(E)=2 Q\left(E^{c}\right)-2 P\left(E^{c}\right)=2 \max \{P(B)-Q(B): B \in \mathcal{B}(\mathbb{S})\} .
$$

Consider the positive part $(P-Q)^{+}$and negative part $(P-Q)^{-}$of $(P-Q)$, that is, $(P-Q)^{+}(B)=$ $(P-Q)(E \cap B)$ and $(P-Q)^{-}(B)=-(P-Q)\left(E^{c} \cap B\right)$ for all $B \in \mathcal{B}(\mathbb{S})$. Both $(P-Q)^{+}$and $(P-Q)^{-}$ are nonnegative finite measures. As follows from (2.4),

$$
\rho_{t v}(P, Q)=2(P-Q)^{+}(E)=2(P-Q)^{-}\left(E^{c}\right) .
$$

The statements of Theorem 2.5 (i,ii) characterize convergence in the total variation via convergence of the values of the measures on open and closed subsets in $\mathbb{S}$. In this respect, these statements are similar to Theorems 2.1 and 2.3, which provide characterizations for weak and setwise convergences. Formula (2.2) indicates that convergence in the total variation can be interpreted as uniform setwise convergence. The same interpretation follows from Theorems 2.3 and 2.5 (i, ii). Theorem 2.5 (iii, iv) indicates that convergence in the total variation can be also interpreted as uniform weak convergence.

Theorem 2.5. The following equalities hold for $P, Q \in \mathbb{P}(\mathbb{S})$ :

(i) $\rho_{t v}(P, Q)=2 \sup \{|P(C)-Q(C)|: C$ is closed in $\mathbb{S}\}=2 \sup \{P(C)-Q(C): C$ is closed in $\mathbb{S}\}$;

(ii) $\rho_{t v}(P, Q)=2 \sup \{|P(\mathcal{O})-Q(\mathcal{O})|: \mathcal{O}$ is open in $\mathbb{S}\}=2 \sup \{P(\mathcal{O})-Q(\mathcal{O}): \mathcal{O}$ is open in $\mathbb{S}\}$;

(iii) $\rho_{t v}(P, Q)=\sup \left\{\int_{\mathbb{S}} f(s) P(d s)-\int_{\mathbb{S}} f(s) Q(d s): f: \mathbb{S} \rightarrow[-1,1]\right.$ is continuous $\}$;

(vi) $\rho_{t v}(P, Q)=\sup \left\{\left|\int_{\mathbb{S}} f(s) P(d s)-\int_{\mathbb{S}} f(s) Q(d s)\right|: f: \mathbb{S} \rightarrow[-1,1]\right.$ is continuous $\}$.

Proof. (i) It is sufficient to show that

$$
\rho_{t v}(P, Q) \leq 2 \sup \{P(C)-Q(C): C \text { is closed in } \mathbb{S}\} .
$$

Since $(P-Q)^{+}$is a measure on a metric space, it is regular; Billingsley [6, Theorem 1.1] or Bogachev [7, Theorem 7.1.7]. Thus, for $E \in \mathcal{B}(\mathbb{S})$ satisfying (2.5) and for each $\varepsilon>0$ there exists a closed subset $C \subseteq \mathbb{S}$ such that $C \subseteq E$ and $2(P-Q)^{+}(E \backslash C)<\varepsilon$. Due to $C \subseteq E$, the equality $(P-Q)(C)=(P-Q)^{+}(C)$ holds. Therefore, in view of (2.5),

$$
\rho_{t v}(P, Q)<2(P-Q)^{+}(C)+\varepsilon \leq 2 \sup \{P(C)-Q(C): C \text { is closed in } \mathbb{S}\}+\varepsilon \text {. }
$$

Since $\varepsilon>0$ is an arbitrary, inequality (2.6) holds.

(ii) Since of $\rho_{t v}(P, Q)=\rho_{t v}(Q, P)$ and

$$
\sup \{P(C)-Q(C): C \text { is closed in } \mathbb{S}\}=\sup \{Q(\mathcal{O})-P(\mathcal{O}): \mathcal{O} \text { is open in } \mathbb{S}\}
$$

(i) implies (ii). 
(iii) In view of (2.3), it is sufficient to show that

$$
\rho_{t v}(P, Q) \leq \sup \left\{\int_{\mathbb{S}} f(s) P(d s)-\int_{\mathbb{S}} f(s) Q(d s): f: \mathbb{S} \rightarrow[-1,1] \text { is continuous }\right\} .
$$

Since the supremum in 2.1) is achieved at the function $f_{E, E^{c}}(s)=\mathbf{I}\{s \in E\}-\mathbf{I}\left\{s \in E^{c}\right\}$,

$$
\rho_{t v}(P, Q)=\int_{\mathbb{S}} f_{E, E^{c}}(s)(P-Q)(d s)
$$

Since of $(P-Q)^{+}$and $(P-Q)^{-}$are measures on a metric space, they are regular; Billingsley [6, Theorem 1.1] or Bogachev [7, Theorem 7.1.7]. Thus, for $E, E^{c} \in \mathcal{B}(\mathbb{S})$ and for each $\varepsilon>0$, there exist closed subsets $C_{1}, C_{2} \subseteq \mathbb{S}$ such that $C_{1} \subseteq E, C_{2} \subseteq E^{c}$, and $(P-Q)^{+}\left(E \backslash C_{1}\right)+(P-Q)^{-}\left(E^{c} \backslash C_{2}\right)<\varepsilon$. Therefore,

$$
\int_{\mathbb{S}} f_{E, E^{c}}(s)(P-Q)(d s) \leq \int_{\mathbb{S}} f_{C_{1}, C_{2}}(s)(P-Q)(d s)+\varepsilon,
$$

where $f_{C_{1}, C_{2}}(s)=\mathbf{I}\left\{s \in C_{1}\right\}-\mathbf{I}\left\{s \in C_{2}\right\}, s \in \mathbb{S}$. Note that the restriction of $f_{C_{1}, C_{2}}$ on a closed subset $C_{1} \cup C_{2}$ in $\mathbb{S}$ is continuous. Since a metric space is a normal topological space, Tietze-UrysohnBrouwer extension theorem implies the existence of a continuous extension of $f_{C_{1}, C_{2}}$ on $\mathbb{S}$, that is, there is a continuous function $\tilde{f}_{C_{1}, C_{2}}: \mathbb{S} \rightarrow[-1,1]$ such that $\tilde{f}_{C_{1}, C_{2}}(s)=f_{C_{1}, C_{2}}(s)$ for any $s \in C_{1} \cup C_{2}$. Thus,

$$
\int_{\mathbb{S}} f_{C_{1}, C_{2}}(s)(P-Q)(d s) \leq \int_{\mathbb{S}} \tilde{f}_{C_{1}, C_{2}}(s)(P-Q)(d s)+\varepsilon .
$$

According to (2.8)-(2.10), for any $\varepsilon>0$

$$
\rho_{t v}(P, Q) \leq \sup \left\{\int_{\mathbb{S}} f(s) P(d s)-\int_{\mathbb{S}} f(s) Q(d s): f: \mathbb{S} \rightarrow[-1,1] \text { is continuous }\right\}+2 \varepsilon,
$$

which yields inequality (2.7).

(iv) According to (iii) and the definition of $\rho_{t v}(P, Q)$,

$$
\begin{aligned}
& \rho_{t v}(P, Q)=\sup \left\{\int_{\mathbb{S}} f(s) P(d s)-\int_{\mathbb{S}} f(s) Q(d s): f: \mathbb{S} \rightarrow[-1,1] \text { is continuous }\right\} \leq \\
& \sup \left\{\left|\int_{\mathbb{S}} f(s) P(d s)-\int_{\mathbb{S}} f(s) Q(d s)\right|: f: \mathbb{S} \rightarrow[-1,1] \text { is continuous }\right\} \leq \rho_{t v}(P, Q),
\end{aligned}
$$

which implies (iv).

For a function $f$ on $\mathbb{R}$, let $V(f)$ denote its total variation. Let $P_{i}, i=1,2$, be probability measures on $\left(\mathbb{R}^{1}, \mathcal{B}\left(\mathbb{R}^{1}\right)\right)$, and $F_{i}(x)=P_{i}\{(-\infty, x]\}, x \in \mathbb{R}^{1}$, be the corresponding distribution functions. The following well-known statement characterizes convergence in the total variation in terms of convergence of distribution functions.

Theorem 2.6. (Cohn [8, Exercise 6, p. 137]). $\rho_{t v}\left(P_{1}, P_{2}\right)=V\left(F_{1}-F_{2}\right)$ for all $P_{1}, P_{2} \in \mathbb{P}\left(\mathbb{R}^{1}\right)$. 


\section{Sufficient Conditions for Weak and Setwice Convergence}

Lemma 3.1. Let $\left\{P_{n}\right\}_{n=1,2, \ldots}$ be a sequence of probability measures from $\mathbb{P}(\mathbb{S})$ and $P \in \mathbb{P}(\mathbb{S})$. If for a measurable subset $B$ of $\mathbb{S}$ there is a countable sequence of measurable subsets $B_{1}, B_{2}, \ldots$ of $B$ such that:

(i) $B=\cup_{i=1}^{\infty} B_{j}$,

(ii) $\liminf \operatorname{in}_{n \rightarrow \infty} P_{n}\left(\cup_{j=1}^{k} B_{j}\right) \geq P\left(\cup_{j=1}^{k} B_{j}\right)$ for all $k=1,2, \ldots$, then

$$
\liminf _{n \rightarrow \infty} P_{n}(B) \geq P(B)
$$

Proof. For an arbitrary $\epsilon>0$ consider an integer $k(\epsilon)$ such that $P\left(\cup_{j=1}^{k(\epsilon)} B_{j}\right) \geq P(B)-\epsilon$. Then

$$
\liminf _{n \rightarrow \infty} P_{n}(B) \geq \liminf _{n \rightarrow \infty} P_{n}\left(\cup_{j=1}^{k(\epsilon)} B_{j}\right) \geq P\left(\cup_{j=1}^{k(\epsilon)} B_{j}\right) \geq P(B)-\epsilon .
$$

Since $\epsilon>0$ is arbitrary, inequality 3.1 holds.

Corollary 3.2. Let $\left\{P_{n}\right\}_{n=1,2, \ldots}$ be a sequence of probability measures from $\mathbb{P}(\mathbb{S})$ and $P \in \mathbb{P}(\mathbb{S})$. If for a each open subset $\mathcal{O}$ of $\mathbb{S}$ there is a countable sequence of measurable subsets $B_{1}, B_{2}, \ldots$ of $\mathcal{O}$ such that:

(i) $\mathcal{O}=\cup_{i=1}^{\infty} B_{j}$,

(ii) $\liminf _{n \rightarrow \infty} P_{n}\left(\cup_{j=1}^{k} B_{j}\right) \geq P\left(\cup_{j=1}^{k} B_{j}\right)$ for all $k=1,2, \ldots$, then then $P_{n} \stackrel{w}{\longrightarrow} P$.

Proof. In view of Lemma $3.1, \liminf _{n \rightarrow \infty} P_{n}(\mathcal{O}) \geq P(\mathcal{O})$ for all open subsets $\mathcal{O}$ of $\mathbb{S}$. In view of Theorem 2.1, this is equivalent to $P_{n} \stackrel{w}{\longrightarrow} P$.

Theorem 3.3. Let $\left\{P_{n}\right\}_{n=1,2, \ldots}$ be a sequence of probability measures from $\mathbb{P}(\mathbb{S})$ and $P \in \mathbb{P}(\mathbb{S})$. If the topology on $\mathbb{S}$ has a countable base $\tau_{b}$, then $P_{n} \stackrel{w}{\longrightarrow} P$ if and only if $\lim _{\inf }{ }_{n \rightarrow \infty} P_{n}\left(\mathcal{O}^{*}\right) \geq P\left(\mathcal{O}^{*}\right)$ for each finite union $\mathcal{O}^{*}=\cup_{i=1}^{k} \mathcal{O}_{i}$ with $\mathcal{O}_{i} \in \tau_{b}, k=1,2, \ldots$.

Proof. Since $P_{n} \stackrel{w}{\longrightarrow} P$ if an only if $\liminf _{n \rightarrow \infty} P_{n}(\mathcal{O}) \geq P(\mathcal{O})$ for each open $\mathcal{O} \subseteq \mathbb{S}$, the necessary condition is obvious. The sufficient part follows from Corollary 3.2 because any open subset $\mathcal{O}$ of $\mathbb{S}$ can be represented as $\mathcal{O}^{*}=\cup_{i=1}^{\infty} \mathcal{O}_{i}$ with $\mathcal{O}_{i} \in \tau_{b}, i=1,2, \ldots$.

Lemma 3.1 can be used to formulate the following criterion for setwise convergence.

Lemma 3.4. Let $\left\{P_{n}\right\}_{n=1,2, \ldots}$ be a sequence of probability measures from $\mathbb{P}(\mathbb{S})$ and $P \in \mathbb{P}(\mathbb{S})$. Then the following statements hold:

(i) Iffor a measurable subset $C$ of $\mathbb{S}$, both sets $B=C$ and $B=C^{c}$, where $C^{c}=\mathbb{S} \backslash C$ is the complement of $C$, satisfy the conditions of Lemma 3.1 then $P_{n}(C) \rightarrow P(C)$.

(ii) If for each open subset $\mathcal{O} \subseteq \mathbb{S}$, both sets $B=\mathcal{O}$ and its complement $B=\mathcal{O}^{c}$ satisfy conditions ( $i$ ) and (ii) of Lemma 3.1 then $P_{n} \stackrel{s}{\longrightarrow} P$.

Proof. (i) Lemma3.1 implies that $\liminf _{n \rightarrow \infty} P_{n}(C) \geq P(C)$ and $\liminf _{n \rightarrow \infty} P_{n}\left(C^{c}\right) \geq P\left(C^{c}\right)$. Since $P$ and $P_{n}, n=1,2, \ldots$ are probability measures, $\lim _{n \rightarrow \infty} P_{n}(C)=P(C)$. (ii) In view of (i), $P_{n}(\mathcal{O}) \rightarrow P(\mathcal{O})$ for each open subset $\mathcal{O}$ of $\mathbb{S}$. In view of Theorem $2.3, P_{n} \stackrel{s}{\longrightarrow} P$. 
For setwise convergence the following theorem states the conditions similar to the conditions of Theorem 3.3 for weak convergence.

Theorem 3.5. Let $\left\{P_{n}\right\}_{n=1,2, \ldots}$ be a sequence of probability measures from $\mathbb{P}(\mathbb{S})$ and $P \in \mathbb{P}(\mathbb{S})$. If the topology on $\mathbb{S}$ has a countable base $\tau_{b}$, then $P_{n} \stackrel{s}{\longrightarrow} P$ if and only if the following two conditions hold:

(i) $\liminf _{n \rightarrow \infty} P_{n}\left(\mathcal{O}^{*}\right) \geq P\left(\mathcal{O}^{*}\right)$ for each finite union $\mathcal{O}^{*}=\cup_{i=1}^{k} \mathcal{O}_{i}$, where $\mathcal{O}_{i} \in \tau_{b}, k=1,2, \ldots$;

(ii) each closed subset $B \subseteq \mathbb{S}$ satisfies conditions ( $i$ ) and (ii) of Lemma 3.1

Proof. Let $\mathcal{O}$ be an arbitrary open subset of $\mathbb{S}$. In view of (i), Theorem 2.1 implies that $\lim \inf _{n \rightarrow \infty} P_{n}(\mathcal{O}) \geq$ $P(\mathcal{O})$. In view of (ii), Lemma 3.1 implies that $\liminf _{n \rightarrow \infty} P_{n}\left(\mathcal{O}^{c}\right) \geq P\left(\mathcal{O}^{c}\right)$. Thus $\lim _{n \rightarrow \infty} P_{n}(\mathcal{O})=$ $P(\mathcal{O})$. Since $\mathcal{O}$ is an arbitrary open subset of $\mathbb{S}$, Theorem 2.3 implies that $P_{n} \stackrel{s}{\longrightarrow} P$.

In some applications, it is more convenient to verify convergence of probabilities for intersections of events than for unions of events. The following lemma links the convergence of probabilities for intersections and unions of events.

Lemma 3.6. Let $\mathcal{L}=\left\{B_{1}, \ldots, B_{N}\right\}$ be a finite collection of measurable subsets of $\mathbb{S}$. Then

$$
\lim _{n \rightarrow \infty} P_{n}\left(\cap_{B_{i} \in \mathcal{L}^{\prime}} B_{i}\right) \rightarrow P\left(\cap_{B_{i} \in \mathcal{L}^{\prime}} B_{i}\right)
$$

for all the subsets $\mathcal{L}^{\prime} \subseteq \mathcal{L}$ if and only if

$$
\lim _{n \rightarrow \infty} P_{n}\left(\cup_{B_{i} \in \mathcal{L}^{\prime}} B_{i}\right) \rightarrow P\left(\cup_{B_{i} \in \mathcal{L}^{\prime}} B_{i}\right)
$$

for all the subsets $\mathcal{L}^{\prime} \subseteq \mathcal{L}$

Proof. If the convergence holds for intersections, it holds for unions because of the inclusion-exclusion principle. If the convergence holds for unions, it holds for intersections because of the inclusion-exclusion principle and induction in the number of sets in $\mathcal{L}$.

The following two statements follow from Corollary 3.2 and Theorem 3.3 respectively.

Corollary 3.7. Let $\left\{P_{n}\right\}_{n=1,2, \ldots}$ be a sequence of probability measures from $\mathbb{P}(\mathbb{S})$ and $P \in \mathbb{P}(\mathbb{S})$. If for a each open subset $\mathcal{O}$ of $\mathbb{S}$ there is a sequence of measurable subsets $B_{1}, B_{2}, \ldots$ of $\mathcal{O}$ such that:

(i) $\mathcal{O}=\cup_{i=1}^{\infty} B_{j}$,

(ii) $\lim _{n \rightarrow \infty} P_{n}\left(\cap_{j=1}^{k} B_{i_{j}}\right)=P\left(\cap_{j=1}^{k} B_{i_{j}}\right)$ for all $\left\{B_{i_{1}}, B_{i_{2}}, \ldots, B_{i_{k}}\right\} \subseteq\left\{B_{1}, B_{2}, \ldots\right\}, k=1,2, \ldots$, then $P_{n} \stackrel{w}{\longrightarrow} P$.

Proof. In view of Lemma 3.6, for each open subset $\mathcal{O}$ of $\mathbb{S}$ condition (ii) implies that $\lim _{n \rightarrow \infty} P_{n}\left(\cup_{j=1}^{k} B_{j}\right)=$ $P\left(\cup_{j=1}^{k} B_{j}\right)$ for all $k=1,2, \ldots$, and according to Corollary 3.2 these equalities imply that $P_{n} \stackrel{w}{\longrightarrow} P$.

Corollary 3.8. Let $\left\{P_{n}\right\}_{n=1,2, \ldots}$ be a sequence of probability measures from $\mathbb{P}(\mathbb{S})$ and $P \in \mathbb{P}(\mathbb{S})$. If the topology on $\mathbb{S}$ has a countable base $\tau_{b}$ such that $P_{n}(\mathcal{O}) \rightarrow P(\mathcal{O})$ for each finite intersection $\mathcal{O}=\cap_{i=1}^{k} \mathcal{O}_{i}$ with $\mathcal{O}_{i} \in \tau_{b}, i=1,2, \ldots, k$, then $P_{n} \stackrel{w}{\longrightarrow} P$. 
Proof. In view of Lemma $3.6 \lim _{n \rightarrow \infty} P_{n}\left(\mathcal{O}^{*}\right)=P\left(\mathcal{O}^{*}\right)$ for each finite union $\mathcal{O}^{*}=\cup_{i=1}^{k} \mathcal{O}_{i}$ with $\mathcal{O}_{i} \in \tau_{b}$, $k=1,2, \ldots$. Theorem 3.3 implies that $P_{n} \stackrel{w}{\longrightarrow} P$.

The following example demonstrates that the assumptions of Corollary 3.8 does not imply that $P_{n} \stackrel{s}{\longrightarrow} P$.

Example 3.9. Let $\mathbb{S}=\mathbb{R}^{1}, P$ be a deterministic measure concentrated at the point $a=\sqrt{2}$, and $P_{n}$ be deterministic measures concentrated at the points $a_{n}=\sqrt{2}+n^{-1}, n=1,2, \ldots$. Since $a_{n} \rightarrow a$, then $P_{n} \stackrel{w}{\longrightarrow} P$ as $n \rightarrow \infty$. Let $\tau_{B}$ be the family consisting of an empty set, $\mathbb{R}^{1}$, and of all the open intervals on $\mathbb{R}^{1}$ with rational ends. Then $\tau_{b}$ is a countable base of the topology on $\mathbb{R}^{1}$ generated by the Euclidean metric. Observe that $\mathcal{O}_{1} \cap \mathcal{O}_{2} \in \tau_{b}$ for all $\mathcal{O}_{1}, \mathcal{O}_{2} \in \tau_{b}$, and $\lim _{n \rightarrow \infty} P_{n}\left(\left(b_{1}, b_{2}\right)\right)=\mathbf{I}\left\{a \in\left(b_{1}, b_{2}\right)\right\}=P\left(\left(b_{1}, b_{2}\right)\right)$, for any rational $b_{1}<b_{2}$. Thus the assumptions of Corollary 3.8 hold. However, of course, it is not true that $P_{n} \stackrel{s}{\longrightarrow} P$, because $P_{n}(\{a\})=0$ for all $n=1,2, \ldots$, but $P(\{a\})=1$.

Corollary 3.10. Let $\left\{P_{n}\right\}_{n=1,2, \ldots}$ be a sequence of probability measures from $\mathbb{P}(\mathbb{S})$ and $P \in \mathbb{P}(\mathbb{S})$. If the topology on $\mathbb{S}$ has a countable base $\tau_{b}$ such that $P_{n}(\mathcal{O}) \rightarrow P(\mathcal{O})$ for each finite intersection $\mathcal{O}=\cap_{i=1}^{k} \mathcal{O}_{i}$ with $\mathcal{O}_{i} \in \tau_{b}, i=1,2, \ldots, k$, and, in addition, for any close set $C \subseteq \mathbb{S}$ there is a sequence of measurable subsets $B_{1}, B_{2}, \ldots$ of $C$ such that $C=\cup_{i=1}^{\infty} B_{j}$ and condition (ii) of Corollary 3.7 holds, then $P_{n} \stackrel{s}{\longrightarrow} P$.

Proof. Let $\mathcal{O}$ be an arbitrary open subset. In view of Corollary 3.8 , the properties of the base $\tau_{b}$ imply that $P_{n} \stackrel{w}{\longrightarrow} P$. Therefore

$$
\liminf _{n \rightarrow \infty} P_{n}(\mathcal{O}) \geq P(\mathcal{O}) .
$$

Let $C=\mathcal{O}^{c}$. Condition (ii) of Corollary 3.7 and Lemma 3.6 imply that $\lim _{n \rightarrow \infty} P_{n}\left(\cup_{j=1}^{k} B_{j}\right)=P\left(\cup_{j=1}^{k} B_{j}\right)$ for all $k=1,2, \ldots$. In view of Lemma 3.1 ,

$$
\liminf _{n \rightarrow \infty} P_{n}\left(\mathcal{O}^{c}\right) \geq P\left(\mathcal{O}^{c}\right) .
$$

Inequalities (3.2) and (3.3) imply that $\lim _{n \rightarrow \infty} P_{n}(\mathcal{O})=P(\mathcal{O})$. Since $\mathcal{O}$ is an arbitrary open subset of $\mathbb{S}$, Theorem 2.3 implies that $P_{n} \stackrel{s}{\longrightarrow} P$.

\section{Continuity of Transition Probabilities}

For a Borel subset $S$ of a metric space $(\mathbb{S}, \rho)$, where $\rho$ is a metric, consider the metric space $(S, \rho)$. A set $B$ is called open (closed, compact) in $S$ if $B \subseteq S$ and $B$ is open (closed, compact) in $(S, \rho)$. Of course, if $S=\mathbb{S}$, we omit "in $\mathbb{S}$ ". Observe that, in general, an open (closed, compact) set in $S$ may not be open (closed, compact). Open sets in $S$ form the topology on $S$ defined by the restriction of metric $\rho$ on $S$.

For metric spaces $\mathbb{S}_{1}$ and $\mathbb{S}_{2}$, a (Borel-measurable) stochastic kernel (sometimes called transition probability) $R\left(d s_{1} \mid s_{2}\right)$ on $\mathbb{S}_{1}$ given $\mathbb{S}_{2}$ is a mapping $R(\cdot \mid \cdot): \mathcal{B}\left(\mathbb{S}_{1}\right) \times \mathbb{S}_{2} \rightarrow[0,1]$, such that $R\left(\cdot \mid s_{2}\right)$ is a probability measure on $\mathbb{S}_{1}$ for any $s_{2} \in \mathbb{S}_{2}$, and $R(B \mid \cdot)$ is a Borel-measurable function on $\mathbb{S}_{2}$ for any Borel set $B \in \mathcal{B}\left(\mathbb{S}_{1}\right)$. A stochastic kernel $R\left(d s_{1} \mid s_{2}\right)$ on $\mathbb{S}_{1}$ given $\mathbb{S}_{2}$ defines a Borel measurable mapping $s_{2} \rightarrow R\left(\cdot \mid s_{2}\right)$ of $\mathbb{S}_{2}$ to the metric space $\mathbb{P}\left(\mathbb{S}_{1}\right)$ endowed with the topology of weak convergence. A stochastic kernel $R\left(d s_{1} \mid s_{2}\right)$ on $\mathbb{S}_{1}$ given $\mathbb{S}_{2}$ is called weakly continuous (setwise continuous, continuous in the total 
variation), if $R\left(\cdot \mid s^{(n)}\right)$ converges weakly (setwise, in the total variation) to $R(\cdot \mid s)$ whenever $s^{(n)}$ converges to $s$ in $\mathbb{S}_{2}$.

In the rest of this section, $\mathbb{S}_{1}, \mathbb{S}_{2}$ and $\mathbb{S}_{3}$ are Borel subsets of Polish (complete separable metric) spaces, and $P$ is a stochastic kernel on $\mathbb{S}_{1} \times \mathbb{S}_{2}$ given $\mathbb{S}_{3}$. The following statement follows from Corollary 3.8 As follows from Lemma 3.6 , the continuity of finite intersection in the condition of Corollary 4.1 can be replaced with the assumption that probabilities of finite unions are continuous.

Corollary 4.1. If the topology on $\mathbb{S}_{i}, i=1,2$, has a countable base $\tau_{b}^{i}$ such that $P\left(\mathcal{O}_{1} \times \mathcal{O}_{2} \mid \cdot\right)$ is continuous on $\mathbb{S}_{3}$ for each finite intersections $\mathcal{O}_{i}=\cap_{j=1}^{N} \mathcal{O}_{i}^{j}$ with $\mathcal{O}_{i}^{j} \in \tau_{b}^{i}, j=1,2, \ldots, N, i=1,2$, then the stochastic kernel $P$ on $\mathbb{S}_{1} \times \mathbb{S}_{2}$ given $\mathbb{S}_{3}$ is weakly continuous.

Proof. Let $\tau_{b}^{1,2}:=\left\{\mathcal{O}_{1}^{\prime} \times \mathcal{O}_{2}^{\prime}: \mathcal{O}_{i}^{\prime} \in \tau_{b}^{i}, i=1,2\right\}$. Note that $\tau_{b}^{1,2}$ is a countable base of the topology on $\mathbb{S}_{1} \times$ $\mathbb{S}_{2}$ defined as the product of the topologies on $\mathbb{S}_{1}$ and $\mathbb{S}_{2}$. Observe that $\cap_{j=1}^{N}\left(\mathcal{O}_{1}^{j} \times \mathcal{O}_{2}^{j}\right)=\left(\cap_{j=1}^{N} \mathcal{O}_{1}^{j}\right) \times$ $\left(\cap_{j=1}^{N} \mathcal{O}_{2}^{j}\right)$ for any finite tuples of open sets $\left\{\mathcal{O}_{i}^{j}\right\}_{j=1}^{N}$ from $\tau_{b}^{i}, i=1,2$. Denote $\mathcal{O}_{i}=\cap_{j=1}^{N} \mathcal{O}_{i}^{j}$ for $i=1,2$. By the assumption of Corollary $4.1 P_{n}\left(\mathcal{O}_{1} \times \mathcal{O}_{2} \mid \cdot\right)$ is continuous on $\mathbb{S}_{3}$. This means that the assumption of Corollary 3.8 holds for the base $\tau_{b}^{1,2}$. Corollary 3.8 implies that the stochastic kernel $P$ on $\mathbb{S}_{1} \times \mathbb{S}_{2}$ given $\mathbb{S}_{3}$ is weakly continuous.

Let $\mathbb{F}(\mathbb{S})$ and $\mathbb{C}(\mathbb{S})$ be respectively the spaces of all real-valued functions and all bounded continuous functions defined on the metric space $\mathbb{S}$. A subset $\mathcal{A}_{0} \subseteq \mathbb{F}(\mathbb{S})$ is said to be equicontinuous at a point $s \in \mathbb{S}$, if $\sup _{f \in \mathcal{A}_{0}}\left|f\left(s^{\prime}\right)-f(s)\right| \rightarrow 0$ as $s^{\prime} \rightarrow s$. If a family $\mathcal{A}_{0} \subseteq \mathbb{F}(\mathbb{S})$ is equicontinuous at each point $s \in \mathbb{S}$, it is called equicontinuous on $\mathbb{S}$. A subset $\mathcal{A}_{0} \subseteq \mathbb{F}(\mathbb{S})$ is said to be uniformly bounded, if there exists a constant $M<+\infty$ such that $|f(s)| \leq M$ for all $s \in \mathbb{S}$ and for all $f \in \mathcal{A}_{0}$. Obviously, if a subset $\mathcal{A}_{0} \subseteq \mathbb{F}(\mathbb{S})$ is equicontinuous at all the points $s \in \mathbb{S}$ and uniformly bounded, then $\mathcal{A}_{0} \subseteq \mathbb{C}(\mathbb{S})$.

Theorem 4.2. (Feinberg et al. [15, Theorem 5.2]). Let $\mathbb{S}_{1}, \mathbb{S}_{2}$, and $\mathbb{S}_{3}$ be arbitrary metric spaces, $P\left(d s_{2} \mid s_{1}\right)$ be a weakly continuous stochastic kernel on $\mathbb{S}_{2}$ given $\mathbb{S}_{1}$, and a subset $\mathcal{A}_{0} \subseteq \mathbb{C}\left(\mathbb{S}_{2} \times \mathbb{S}_{3}\right)$ be equicontinuous at all the points $\left(s_{2}, s_{3}\right) \in \mathbb{S}_{2} \times \mathbb{S}_{3}$ and uniformly bounded. If $\mathbb{S}_{2}$ is separable, then for every open set $\mathcal{O}$ in $\mathbb{S}_{2}$ the family of functions defined on $\mathbb{S}_{1} \times \mathbb{S}_{3}$,

$$
\mathcal{A}_{\mathcal{O}}=\left\{\left(s_{1}, s_{3}\right) \rightarrow \int_{\mathcal{O}} f\left(s_{2}, s_{3}\right) P\left(d s_{2} \mid s_{1}\right): f \in \mathcal{A}_{0}\right\},
$$

is equicontinuous at all the points $\left(s_{1}, s_{3}\right) \in \mathbb{S}_{1} \times \mathbb{S}_{3}$ and uniformly bounded.

Further as $\tau(\mathbb{S})$ we denote the family of all open subsets of a metric space $\mathbb{S}$. For each $B \in \mathcal{B}\left(\mathbb{S}_{1}\right)$ consider a family of functions

$$
\mathcal{P}_{B}=\left\{s_{3} \rightarrow P\left(B \times C \mid s_{3}\right): C \in \tau\left(\mathbb{S}_{2}\right)\right\}
$$

mapping $\mathbb{S}_{3}$ into $[0,1]$.

Lemma 4.3. Let $B \in \mathcal{B}\left(\mathbb{S}_{1}\right)$. The family of functions $\mathcal{P}_{B}$ is equicontinuous at a point $s_{3} \in \mathbb{S}_{3}$ if and only if

$$
\sup _{C \in \mathcal{B}\left(\mathbb{S}_{2}\right)}\left|P\left(B \times C \mid s_{3}^{(n)}\right)-P\left(B \times C \mid s_{3}\right)\right| \rightarrow 0 \quad \text { as } \quad s_{3}^{(n)} \rightarrow s_{3} .
$$


Proof. According to the definition of the equicontinuity of the family of functions $\mathcal{P}_{B}$ at a point, it is sufficient to prove that (4.1) follows from

$$
\sup _{C \in \tau\left(\mathbb{S}_{2}\right)}\left|P\left(B \times C \mid s_{3}^{(n)}\right)-P\left(B \times C \mid s_{3}\right)\right| \rightarrow 0 \quad \text { as } \quad s_{3}^{(n)} \rightarrow s_{3} .
$$

Indeed, if $P\left(B \times \mathbb{S}_{2} \mid s_{3}\right)=0$, then $\sup _{C \in \mathcal{B}\left(\mathbb{S}_{2}\right)}\left|P\left(B \times C \mid s_{3}^{(n)}\right)-P\left(B \times C \mid s_{3}\right)\right|=P\left(B \times \mathbb{S}_{2} \mid s_{3}^{(n)}\right) \rightarrow$ $P\left(B \times \mathbb{S}_{2} \mid s_{3}\right)=0$ as $s_{3}^{(n)} \rightarrow s_{3}$, because $\mathbb{S}_{2} \in \tau\left(\mathbb{S}_{2}\right)$. Otherwise, when $P\left(B \times \mathbb{S}_{2} \mid s_{3}\right)>0$, according to the convergence $P\left(B \times \mathbb{S}_{2} \mid s_{3}^{(n)}\right) \rightarrow P\left(B \times \mathbb{S}_{2} \mid s_{3}\right)>0$ as $s_{3}^{(n)} \rightarrow s_{3}$, Theorem 2.5 (ii) applied to the probability measures $C \rightarrow P\left(B \times C \mid s_{3}^{(n)}\right) / P\left(B \times \mathbb{S}_{2} \mid s_{3}^{(n)}\right)$ and $C \rightarrow P\left(B \times C \mid s_{3}\right) / P\left(B \times \mathbb{S}_{2} \mid s_{3}\right)$ from $\mathbb{P}\left(\mathbb{S}_{2}\right)$, where $n$ is rather large, yields that 4.1 holds, that is, the family of functions $\mathcal{P}_{B}$ is equicontinuous at a point $s_{3} \in \mathbb{S}_{3}$.

Let $P^{\prime}$ be the marginal of $P$ on $\mathbb{S}_{2}$, that is, $P^{\prime}\left(C \mid s_{3}\right):=P\left(\mathbb{S}_{1} \times C \mid s_{3}\right), C \in \mathcal{B}\left(\mathbb{S}_{2}\right), s_{3} \in \mathbb{S}_{3}$. There exists a stochastic kernel $H$ on $\mathbb{S}_{1}$ given $\mathbb{S}_{2} \times \mathbb{S}_{3}$ such that, for all $B \in \mathcal{B}\left(\mathbb{S}_{1}\right), C \in \mathcal{B}\left(\mathbb{S}_{2}\right), s_{3} \in \mathbb{S}_{3}$

$$
P\left(B \times C \mid s_{3}\right)=\int_{C} H\left(B \mid s_{2}, s_{3}\right) P^{\prime}\left(d s_{2} \mid s_{3}\right) ;
$$

Bertsekas and Shreve [5, Proposition 7.27]. Moreover, for each $s_{3} \in \mathbb{S}_{3}$, the distribution $H\left(\cdot \mid s_{2}, s_{3}\right)$ is $P^{\prime}\left(\cdot \mid s_{3}\right)$-a.s. unique in $s_{2}$, that is, if $H_{1}$ and $H_{2}$ satisfy (4.2) then $P^{\prime}\left(C^{*} \mid s_{3}\right)=0$, where $C^{*}:=\left\{s_{2} \in \mathbb{S}_{2}\right.$ : $H_{1}\left(B \mid s_{2}, s_{3}\right) \neq H_{2}\left(B \mid s_{2}, s_{3}\right)$ for some $\left.B \in \mathcal{B}\left(\mathbb{S}_{1}\right)\right\}$; Bertsekas and Shreve [5, Corollary 7.27.1].

Theorem 4.4. Let the topology on $\mathbb{S}_{1}$ have a countable base $\tau_{b}$ satisfying the following two conditions:

(i) $\mathbb{S}_{1} \in \tau_{b}$,

(ii) for each finite intersection $\mathcal{O}=\bigcap_{i=1}^{N} \mathcal{O}^{i}$ of sets $\mathcal{O}^{i} \in \tau_{b}, i=1,2, \ldots, N$, the family of functions $\mathcal{P}_{\mathcal{O}}$ is equicontinuous at a point $s_{s} \in \mathbb{S}_{3}$.

Then, for any sequence $\left\{s_{3}^{(n)}\right\}_{n=1,2, \ldots}$ from $\mathbb{S}_{3}$ converging to $s_{3} \in \mathbb{S}_{3}$, there exists a subsequence $\left\{n_{k}\right\}_{k=1,2, \ldots}$ and a set $C^{*} \in \mathcal{B}\left(\mathbb{S}_{2}\right)$ such that

$$
P^{\prime}\left(C^{*} \mid s_{3}\right)=1 \text { and } H\left(\cdot \mid s_{2}, s_{3}^{\left(n_{k}\right)}\right) \text { converges weakly to } H\left(\cdot \mid s_{2}, s_{3}\right) \text { for all } s_{2} \in C^{*} \text { as } k \rightarrow \infty \text {. }
$$

Remark 4.5. According to Lemma 3.6 a countable base $\tau_{b}$ in Theorem 4.4 can be assumed to be closed with respect to the finite unions instead of finite intersections.

Theorem 4.4 implies the following two corollaries. The proof of Theorem 4.4 is provided after the proof of Lemma 4.9

Corollary 4.6. If for each open subset $\mathcal{O}$ of $\mathbb{S}_{1}$ the family of functions $\mathcal{P}_{\mathcal{O}}$ is equicontinuous at a point $s_{3} \in \mathbb{S}_{3}$, then for any sequence $\left\{s_{3}^{(n)}\right\}_{n=1,2, \ldots}$ from $\mathbb{S}_{3}$, that converges to $s_{3} \in \mathbb{S}_{3}$, there exists a subsequence $\left\{n_{k}\right\}_{k=1,2, \ldots}$ and a set $C^{*} \in \mathcal{B}\left(\mathbb{S}_{2}\right)$ such that (4.3) holds.

Proof. The statement of the corollary follows immediately from Theorem 4.4 Indeed, the family of functions $\mathcal{P}_{\mathcal{O}}$ is equicontinuous on $\mathbb{S}_{3}$ for each open set $\mathcal{O}$ of $\mathbb{S}_{1}$. Since $\mathbb{S}_{1}$ is a separable metric space, each countable base of the topology on $\mathbb{S}_{1}$ satisfies assumptions of Theorem 4.4 
Observe that for a stochastic kernel $P$ on $\mathbb{S}_{1} \times \mathbb{S}_{2}$ given $\mathbb{S}_{3}$, equicontinuity at a point $s_{3} \in \mathbb{S}_{3}$ of the family of functions $\mathcal{P}_{\mathcal{O}}$ for all open subsets $\mathcal{O}$ in $\mathbb{S}_{1}$ is a weaker assumption than continuity in the total variation of $P$ on $\mathbb{S}_{1} \times \mathbb{S}_{2}$ given $\mathbb{S}_{3}$ at the point $s_{3}$. Equicontinuiuty of the family of functions $\mathcal{P}_{\mathbb{S}_{1}}$ at a point $s_{3} \in \mathbb{S}_{3}$ is equivalent to the continuity in the total variation of the stochastic kernel $P^{\prime}$ on $\mathbb{S}_{2}$ given $\mathbb{S}_{3}$ at the point $s_{3}$.

Corollary 4.7. Let assumptions of Theorem 4.4 hold. If the setwise convergence takes place in (4.3) instead of the weak convergence, then the stochastic kernel Pon $\mathbb{S}_{1} \times \mathbb{S}_{2}$ given $\mathbb{S}_{3}$ is setwise continuous.

Proof. According to Theorem 2.3 , if the stochastic kernel $P$ on $\mathbb{S}_{1} \times \mathbb{S}_{2}$ given $\mathbb{S}_{3}$ is not setwise continuous, then there exist $\varepsilon>0$, a nonempty open subset $\mathcal{O}$ of $\mathbb{S}_{1} \times \mathbb{S}_{2}$, and a sequence $\left\{s_{3}^{(n)}\right\}_{n=1,2, \ldots}$ that converges to some $s_{3} \in \mathbb{S}_{3}$ such that

$$
\left|P\left(\mathcal{O} \mid s_{3}^{(n)}\right)-P\left(\mathcal{O} \mid s_{3}\right)\right| \geq \varepsilon \text { for each } n=1,2, \ldots .
$$

Let $\mathcal{O}_{2}$ be the projection of $\mathcal{O}$ on $\mathbb{S}_{2}$ and $\mathcal{O}_{\left(s_{2}\right)}:=\left\{s_{1} \in \mathbb{S}_{1}:\left(s_{1}, s_{2}\right) \in \mathcal{O}\right\}$ be the cut of $\mathcal{O}$ at $s_{2} \in \mathcal{O}_{2}$. Since $\mathcal{O}$ is an open set, the sets $\mathcal{O}_{2}$ and $\mathcal{O}_{\left(s_{2}\right)}$ are open. Since $P^{\prime}\left(d s_{2} \mid s_{3}^{(n)}\right)$ converges in the total variation to $P^{\prime}\left(d s_{2} \mid s_{3}\right)$, for any $s_{3} \in \mathbb{S}_{3}$

$$
\left|\int_{\mathcal{O}_{2}} H\left(\mathcal{O}_{\left(s_{2}\right)} \mid s_{2}, s_{3}^{(n)}\right) P^{\prime}\left(d s_{2} \mid s_{3}^{(n)}\right)-\int_{\mathcal{O}_{2}} H\left(\mathcal{O}_{\left(s_{2}\right)} \mid s_{2}, s_{3}^{(n)}\right) P^{\prime}\left(d s_{2} \mid s_{3}\right)\right| \rightarrow 0 \text { as } n \rightarrow \infty .
$$

According to the assumptions of Corollary 4.7 there exists a set $C^{*} \in \mathcal{B}\left(\mathbb{S}_{2}\right)$ and a subsequence $\left\{s_{3}^{\left(n_{k}\right)}\right\}_{k=1,2, \ldots}$ of $\left\{s_{3}^{(n)}\right\}_{n=1,2, \ldots}$ such that $P^{\prime}\left(C^{*} \mid s_{3}\right)=1$ and $H\left(\cdot \mid s_{2}, s_{3}^{\left(n_{k}\right)}\right)$ converges setwise to $H\left(\cdot \mid s_{2}, s_{3}\right)$ for any $s_{2} \in C^{*}$. In particular, $H\left(\mathcal{O}_{\left(s_{2}\right)} \mid s_{2}, s_{3}^{\left(n_{k}\right)}\right) \rightarrow H\left(\mathcal{O}_{\left(s_{2}\right)} \mid s_{2}, s_{3}\right)$ for any $s_{2} \in C^{*}$. Therefore, the dominated convergence theorem yields

$$
\int_{\mathcal{O}_{2}}\left|H\left(\mathcal{O}_{\left(s_{2}\right)} \mid s_{2}, s_{3}^{\left(n_{k}\right)}\right)-H\left(\mathcal{O}_{\left(s_{2}\right)} \mid s_{2}, s_{3}\right)\right| P^{\prime}\left(d s_{2} \mid s_{3}\right) \rightarrow 0 \text { as } k \rightarrow \infty .
$$

Formulae (4.5) and (4.6) imply that as $k \rightarrow \infty$

$$
P\left(\mathcal{O} \mid s_{3}^{\left(n_{k}\right)}\right)=\int_{\mathcal{O}_{2}} H\left(\mathcal{O}_{\left(s_{2}\right)} \mid s_{2}, s_{3}^{\left(n_{k}\right)}\right) P^{\prime}\left(d s_{2} \mid s_{3}^{(n)}\right) \rightarrow \int_{\mathcal{O}_{2}} H\left(\mathcal{O}_{\left(s_{2}\right)} \mid s_{2}, s_{3}\right) P^{\prime}\left(d s_{2} \mid s_{3}\right)=P\left(\mathcal{O} \mid s_{3}\right) .
$$

This contradicts (4.4). Thus the stochastic kernel $P$ on $\mathbb{S}_{1} \times \mathbb{S}_{2}$ given $\mathbb{S}_{3}$ is setwise continuous.

The proof of Theorem 4.4 uses several auxiliary results.

Lemma 4.8. (Feinberg et. al [15, Theorem 5.5]). Let $h$ and $\left\{h^{(n)}\right\}_{n=1,2, \ldots}$ be Borel-measurable uniformly bounded real-valued functions defined on a metric space $\mathbb{S}$ and let $\left\{\mu^{(n)}\right\}_{n=1,2, \ldots}$ be a sequence of probability measures from $\mathbb{P}(\mathbb{S})$ that converge in the total variation to the measure $\mu \in \mathbb{P}(\mathbb{S})$. If

$$
\sup _{C \in \mathcal{B}(\mathbb{S})}\left|\int_{C} h^{(n)}(s) \mu^{(n)}(d s)-\int_{C} h(s) \mu(d s)\right| \rightarrow 0 \quad \text { as } \quad n \rightarrow \infty,
$$

then $\left\{h^{(n)}\right\}_{n=1,2, \ldots}$ converges in probability $\mu$ to $h$ as $n \rightarrow \infty$, and therefore there is a subsequence $\left\{n_{k}\right\}_{k=1,2, \ldots}$ such that $\left\{h^{\left(n_{k}\right)}\right\}_{k=1,2, \ldots}$ converges $\mu$-almost surely to $h$. 
Let $\mathbb{A}_{1}$ be the family of all subsets of $\mathbb{S}_{1}$ that are finite unions of sets from the countable base $\tau_{b}$ of the topology on $\mathbb{S}_{1}$ satisfying the conditions of Theorem 4.4 and $\mathbb{A}_{2}$ be the family of all subsets $B$ of $\mathbb{S}_{1}$ such that $B=\tilde{\mathcal{O}} \backslash \mathcal{O}^{\prime}$ with $\tilde{\mathcal{O}} \in \tau_{b}$ and $\mathcal{O}^{\prime} \in \mathbb{A}_{1}$.

Lemma 4.9. Let the assumptions of Theorem 4.4 hold for some $s_{3} \in \mathbb{S}_{3}$. Then, for any subset $B \in \mathbb{A}_{2}$, the family of functions $\mathcal{P}_{B}$ is equicontinuous at the point $s_{3} \in \mathbb{S}_{3}$.

Proof. Fix an arbitrary $s_{3} \in \mathbb{S}_{3}$. Observe that, if for all $\mathcal{O} \in \mathbb{A}_{1}$ the family of functions $\mathcal{P}_{\mathcal{O}}$ is equicontinuous at the point $s_{3} \in \mathbb{S}_{3}$, then for any subset $B=\tilde{\mathcal{O}} \backslash \mathcal{O}^{\prime}$ of $\mathbb{S}_{1}$ with $\tilde{\mathcal{O}} \in \tau_{b}$ and $\mathcal{O}^{\prime} \in \mathbb{A}_{1}$, the family of functions $\mathcal{P}_{B}$ is equicontinuous at the point $s_{3} \in \mathbb{S}_{3}$. Indeed, according to Lemma 4.3 , for all $s_{3}, s_{3}^{(n)} \in \mathbb{S}_{3}$, $n=1,2, \ldots$, such that $s_{3}^{(n)} \rightarrow s_{3}$ as $n \rightarrow \infty$,

$$
\begin{aligned}
& \sup _{C \in \mathcal{B}\left(\mathbb{S}_{2}\right)}\left|P\left(B \times C \mid s_{3}^{(n)}\right)-P\left(B \times C \mid s_{3}\right)\right|=\sup _{C \in \mathcal{B}\left(\mathbb{S}_{1}\right)}\left|P\left(\left(\tilde{\mathcal{O}} \backslash \mathcal{O}^{\prime}\right) \times C \mid s_{3}^{(n)}\right)-P\left(\left(\tilde{\mathcal{O}} \backslash \mathcal{O}^{\prime}\right) \times C \mid s_{3}\right)\right| \\
\leq & \sup _{C \in \mathcal{B}\left(\mathbb{S}_{2}\right)}\left|P\left(\mathcal{O}^{\prime} \times C \mid s_{3}^{(n)}\right)-P\left(\mathcal{O}^{\prime} \times C \mid s_{3}\right)\right|+\sup _{C \in \mathcal{B}\left(\mathbb{S}_{2}\right)}\left|P\left(\left(\tilde{\mathcal{O}} \cup \mathcal{O}^{\prime}\right) \times C \mid s_{3}^{(n)}\right)-P\left(\left(\tilde{\mathcal{O}} \cup \mathcal{O}^{\prime}\right) \times C \mid s_{3}\right)\right| .
\end{aligned}
$$

The above inequality, the assumption that (4.1) holds for all $\mathcal{O} \in \mathbb{A}_{1}$ and for all $s_{3}, s_{3}^{(n)} \in \mathbb{S}_{3}, n=1,2, \ldots$, such that $s_{3}^{(n)} \rightarrow s_{3}$ as $n \rightarrow \infty$, and the property that if $\mathcal{O}^{\prime} \in \mathbb{A}_{1}$ then $\tilde{\mathcal{O}} \cup \mathcal{O}^{\prime} \in \mathbb{A}_{1}$ for all $\tilde{\mathcal{O}} \in \tau_{b}$ imply that (4.1) holds for any subset $B \in \mathbb{A}_{2}$, that is, the family of functions $\mathcal{P}_{B}$ is equicontinuous at the point $s_{3} \in \mathbb{S}_{3}$. The rest of the proof establishes that, for each $\mathcal{O} \in \mathbb{A}_{1}$, the family of functions $\mathcal{P}_{\mathcal{O}}$ is equicontinuous at the point $s_{3} \in \mathbb{S}_{3}$.

Let $\tau_{b}=\left\{\mathcal{O}^{(j)}\right\}_{j=1,2, \ldots}$. Consider an arbitrary $\mathcal{O} \in \mathbb{A}_{1}$. Then $\mathcal{O}=\cup_{i=1}^{N} \mathcal{O}^{\left(j_{i}\right)}$ for some $N=1,2, \ldots$, where $\mathcal{O}^{\left(j_{i}\right)} \in \tau_{b}, i=1,2, \ldots, N$. Let $\mathbb{A}^{N}=\left\{\cap_{m=1}^{k} \mathcal{O}^{\left(i_{m}\right)}:\left\{i_{1}, i_{2}, \ldots, i_{k}\right\} \subseteq\left\{j_{1}, j_{2}, \ldots j_{N}\right\}\right\}$ be the finite set of possible intersections of $\mathcal{O}^{\left(j_{1}\right)}, \ldots, \mathcal{O}^{\left(j_{N}\right)}$. The principle of inclusion-exclusion implies that for $\mathcal{O}=\cup_{i=1}^{N} \mathcal{O}^{\left(j_{i}\right)}, C \in \mathbb{S}_{2}$, and $s_{3}, s_{3}^{(n)} \in \mathbb{S}_{3}$,

$$
\left|P\left(\mathcal{O} \times C \mid s_{3}\right)-P\left(\mathcal{O} \times C \mid s_{3}^{(n)}\right)\right| \leq \sum_{D \in \mathbb{A}^{N}}\left|P\left(D \times C \mid s_{3}\right)-P\left(D \times C \mid s_{3}^{(n)}\right)\right| .
$$

The above inequality and the assumption of Theorem 4.4 regarding finite intersections of the elements of the base $\tau_{b}$ imply that, for each $\mathcal{O} \in \mathbb{A}_{1}$, the family of functions $\mathcal{P}_{\mathcal{O}}$ is equicontinuous at the point $s_{3} \in \mathbb{S}_{3}$.

Proof of Theorem 4.4 Let $\left\{s_{3}^{(n)}\right\}_{n=1,2, \ldots}$ be a sequence from $\mathbb{S}_{3}$ that converges to $s_{3} \in \mathbb{S}_{3}$. According to Theorem 2.1 (4.3) holds if there exists a subsequence $\left\{n_{m}\right\}_{m=1,2, \ldots}$ and a set $C^{*} \in \mathcal{B}\left(\mathbb{S}_{2}\right)$ such that for all open subsets $\mathcal{O}$ in $\mathbb{S}_{1}$

$$
P^{\prime}\left(C^{*} \mid s_{3}\right)=1 \quad \text { and } \quad \liminf _{m \rightarrow \infty} H\left(\mathcal{O} \mid s_{2}, s_{3}^{\left(n_{m}\right)}\right) \geq H\left(\mathcal{O} \mid s_{2}, s_{3}\right) \quad \text { for all } \quad s_{2} \in C^{*}
$$

The rest of the proof establishes the existence of a subsequence $\left\{s_{3}^{\left(n_{m}\right)}\right\}_{m=1,2, \ldots}$ of the sequence $\left\{s_{3}^{(n)}\right\}_{n=1,2, \ldots}$ and a set $C^{*} \in \mathcal{B}\left(\mathbb{S}_{2}\right)$ such that (4.8) holds for each open subset $\mathcal{O}$ of $\mathbb{S}_{1}$.

Let $\mathbb{A}_{1}$ and $\mathbb{A}_{2}$ be the families of subsets of $\mathbb{S}_{1}$ as defined before Lemma 4.9. Observe that: (i) both $\mathbb{A}_{1}$ and $\mathbb{A}_{2}$ are countable, (ii) every open subset $\mathcal{O}$ of $\mathbb{S}_{1}$ can be represented as

$$
\mathcal{O}=\bigcup_{j=1,2, \ldots} \mathcal{O}^{(j, 1)}=\bigcup_{j=1,2, \ldots} B^{(j, 1)}, \quad \text { for some } \quad \mathcal{O}^{(j, 1)} \in \tau_{b}, j=1,2, \ldots
$$


where $B^{(j, 1)}=\mathcal{O}^{(j, 1)} \backslash\left(\cup_{i=1}^{j-1} \mathcal{O}^{(i, 1)}\right)$ are disjoint elements of $\mathbb{A}_{2}$ (it is allowed that $\mathcal{O}^{(j, 1)}=\emptyset$ or $B^{(j, 1)}=\emptyset$ for some $j=1,2, \ldots)$.

To prove (4.8) for all open subsets $\mathcal{O}$ of $\mathbb{S}_{1}$, we first show that (4.8) holds for all $\mathcal{O} \in \mathbb{A}_{2}$. From Lemmas 4.3, 4.9 and 4.2),

$$
\lim _{n \rightarrow \infty} \sup _{C \in \mathcal{B}\left(\mathbb{S}_{2}\right)}\left|\int_{C} H\left(B \mid s_{2}, s_{3}^{(n)}\right) P^{\prime}\left(d s_{2} \mid s_{3}^{(n)}\right)-\int_{C} H\left(B \mid s_{2}, s_{3}\right) P^{\prime}\left(d s_{2} \mid s_{3}\right)\right|=0, \quad B \in \mathbb{A}_{2} .
$$

Since the set $\mathbb{A}_{2}$ is countable, let $\mathbb{A}_{2}:=\left\{B^{(j)}: j=1,2, \ldots\right\}$. Choose a subsequence $\left\{s_{3}^{\left(n_{k}\right)}\right\}_{k=1,2, \ldots}$ of the sequence $\left\{s_{3}^{(n)}\right\}_{n=1,2, \ldots}$. Denote $s^{(n, 0)}=s_{3}^{(n)}$ for all $n=1,2, \ldots$. For $j=1,2, \ldots$, from (4.10), Lemma 4.8, applied with $s=s_{2}, h^{(n)}(s)=H\left(B^{(j)} \mid s_{2}, s^{(n, j-1)}\right), \mu^{(n)}(\cdot)=P^{\prime}\left(\cdot \mid s^{(n, j-1)}\right)$, $h(s)=H\left(B^{(j)} \mid s_{2}, s_{3}\right)$, and $\mu(\cdot)=P^{\prime}\left(\cdot \mid s_{3}\right)$, there exists a subsequence $\left\{s^{(n, j)}\right\}_{n=1,2, \ldots}$ of the sequence $\left\{s^{(n, j-1)}\right\}_{n=1,2, \ldots}$ and a set $C_{j}^{*} \in \mathcal{B}\left(\mathbb{S}_{2}\right)$ such that

$$
\lim _{n \rightarrow \infty} H\left(B^{(j)} \mid s_{2}, s^{(n, j)}\right)=H\left(B^{(j)} \mid s_{2}, s_{3}\right) \quad \text { for all } \quad s_{2} \in C_{j}^{*}
$$

Let $C^{*}=\cap_{j=1,2, \ldots} C_{j}^{*}$. Observe that $P^{\prime}\left(C^{*} \mid s_{3}\right)=1$. Let $s_{3}^{\left(n_{m}\right)}=s^{(m, m)}, m=1,2, \ldots$. As follows from Cantor's diagonal argument, (4.8) holds with $\mathcal{O}=B^{(j)}$ for all $j=1,2, \ldots$. In other words, (4.8) is proved for all $\mathcal{O} \in \mathbb{A}_{2}$.

Let $\mathcal{O}$ be an arbitrary open set in $\mathbb{S}_{1}$ and $B^{(1,1)}, B^{(2,1)}, \ldots$ be disjoint elements of $\mathbb{A}_{2}$ satisfying [4.9). Then the countable additivity of probability measures implies that, for all $s_{2} \in C^{*}$,

$$
\begin{aligned}
\liminf _{m \rightarrow \infty} H\left(\mathcal{O} \mid s_{2}, s_{3}^{\left(n_{m}\right)}\right) & =\liminf _{m \rightarrow \infty} \sum_{j=1,2, \ldots} H\left(B^{(j, 1)} \mid s_{2}, s_{3}^{\left(n_{m}\right)}\right) \geq \sum_{j=1,2, \ldots} \liminf _{m \rightarrow \infty} H\left(B^{(j, 1)} \mid s_{2}, s_{3}^{\left(n_{m}\right)}\right) \\
& =\sum_{j=1,2, \ldots} H\left(B^{(j, 1)} \mid s_{2}, s_{3}\right)=H\left(\mathcal{O} \mid s_{2}, s_{3}\right) .
\end{aligned}
$$

Therefore, (4.8) holds for all open subsets $\mathcal{O}$ in $\mathbb{S}_{1}$.

Example 4.10. (Stochastic kernel $P$ on $\mathbb{S}_{1} \times \mathbb{S}_{2}$ given $\mathbb{S}_{3}$ satisfies assumptions of Theorem 4.4 but it is not setwise continuous and it does not satisfy the assumption of Corollary 4.6) Let $\mathbb{S}_{1}=\mathbb{R}^{1}, \mathbb{S}_{2}=\{1\}$, $\mathbb{S}_{3}=\left\{1^{-1}, 2^{-1}, \ldots, 0\right\}, \tau_{B}$ be the family consisting of an empty set, $\mathbb{R}^{1}$, and of all the open intervals on $\mathbb{R}^{1}$ with rational ends, and $P\left(B \times C \mid s_{3}\right)=\mathbf{I}\left\{\sqrt{2}+s_{3} \in B\right\} \mathbf{I}\{1 \in C\}, B \in \mathcal{B}\left(\mathbb{S}_{1}\right), C \in \mathcal{B}\left(\mathbb{S}_{2}\right)$. Then $P^{\prime}(C)=\mathbf{I}\{1 \in C\}, H\left(B \mid s_{2}, s_{3}\right)=\mathbf{I}\left\{\sqrt{2}+s_{3} \in B\right\}, B \in \mathcal{B}\left(\mathbb{S}_{1}\right), C \in \mathcal{B}\left(\mathbb{S}_{2}\right)$. Let $\tau_{b}$ be the countable base of the topology on $\mathbb{R}^{1}$ generated by the Euclidean metric described in Example 3.9. The family $\tau_{b}$ is closed under finite intersections, and for any $\mathcal{O} \in \tau_{b}$ the family of functions $\mathcal{P}_{\mathcal{O}}$ is equicontinuous at all the points $s_{3} \in \mathbb{S}_{3}$. Therefore, assumptions of Theorem 4.4 hold.

Note that the function $P\left(B \times C \mid s_{3}\right)$ is not continuous at the point $s_{3}=0$, when $B=\mathbb{R}^{1} \backslash\{\sqrt{2}\}$ and $C=\mathbb{S}_{3}$. Therefore, the family $\mathcal{P}_{B}$ is not equicontinuous at the point $s_{3}=0$, and the assumption of Corollary 4.6 do not hold. Moreover, the sequence $\left\{H\left(B \mid 1, \frac{1}{n}\right)\right\}_{n=1,2, \ldots}$ (and any its subsequence) does not converge to $H(B \mid 1,0)$ and, therefore, the setwise convergence assumption from Corollary 4.7 do not hold. 


\section{Partially Observable Markov Decision Processes}

Convergence properties of probability measures and relevant continuity properties of transition probabilities are broadly used in mathematical methods of stochastic control. In this section, we describe the results for a Bayesian sequential decision model, a POMDP. For POMDPs, posterior probabilities of states of the process form sufficient statistics; see e.g., Hernández-Lerma [17, p. 89]. In terms of Markov Decision Processes, this well-known fact means that it is possible to construct an MDP, called a Completely Observable Markov Decision Process (COMDP), whose state space is the space of probability measures on the original state space. If an optimal policy is found for a COMDP, it is easy to compute an optimal policy for the original POMDP. However, except the cases of finite state spaces (Smallwood and Sondik [31], Sondik [32]), MDMIIs with transition probabilities having densities (Rieder [25], Bäuerle and Rieder [3, Chapter 5]), models explicitly defined by equations for continuous random variables (Striebel [33], Bensoussan [4]), and numerous particular problems studied in the literature, until recently very little had been known about the existence and characterizations of optimal policies for POMDPs and their COMDPs. The main difficulty is that the transition probability for a COMDP is defined via the Bayes formula presented in formula (5.4) below, and the explicit forms of the Bayes formula are known either for discrete events or for continuous random variables; see Shityaev [30, p. 231]. Recently Feinberg et al. [15] established sufficient conditions for the existence of optimal policies and their characterization for POMDPs with Borel state, action, and observation spaces.

In this section we define POMDPs, explain their reduction to COMDPs, survey some of the results from Feinberg et al. [15], and present the condition on joint distributions of posterior distributions and observations that implies weak continuity of transition probabilities for the COMDP. In the following section, we describe a more particular model, the MDMII, and apply Corollary 4.7 and results of this section to it.

Let $\mathbb{X}, \mathbb{Y}$, and $\mathbb{A}$ be Borel subsets of Polish spaces, $P\left(d x^{\prime} \mid x, a\right)$ be a stochastic kernel on $\mathbb{X}$ given $\mathbb{X} \times \mathbb{A}$, $Q(d y \mid a, x)$ be a stochastic kernel on $\mathbb{Y}$ given $\mathbb{A} \times \mathbb{X}, Q_{0}(d y \mid x)$ be a stochastic kernel on $\mathbb{Y}$ given $\mathbb{X}, p$ be a probability distribution on $\mathbb{X}, c: \mathbb{X} \times \mathbb{A} \rightarrow \overline{\mathbb{R}}^{1}=\mathbb{R}^{1} \cup\{+\infty\}$ be a bounded below Borel function on $\mathbb{X} \times \mathbb{A}$.

A $P O M D P$ is specified by a tuple $(\mathbb{X}, \mathbb{Y}, \mathbb{A}, P, Q, c)$, where $\mathbb{X}$ is the state space, $\mathbb{Y}$ is the observation set, $\mathbb{A}$ is the action set, $P\left(d x^{\prime} \mid x, a\right)$ is the state transition law, $Q(d y \mid a, x)$ is the observation stochastic kernel, $c: \mathbb{X} \times \mathbb{A} \rightarrow \overline{\mathbb{R}}^{1}$ is the one-step cost.

The partially observable Markov decision process evolves as follows: (i) at time $t=0$, the initial unobservable state $x_{0}$ has a given prior distribution $p$; (ii) the initial observation $y_{0}$ is generated according to the initial observation stochastic kernel $Q_{0}\left(\cdot \mid x_{0}\right)$; (iii) at each time epoch $t=0,1, \ldots$, if the state of the system is $x_{t} \in \mathbb{X}$ and the decision-maker chooses an action $a_{t} \in \mathbb{A}$, then the cost $c\left(x_{t}, a_{t}\right)$ is incurred; (iv) the system moves to a state $x_{t+1}$ according to the transition law $P\left(\cdot \mid x_{t}, a_{t}\right), t=0,1, \ldots ;(\mathrm{v})$ an observation $y_{t+1} \in \mathbb{Y}$ is generated by the observation stochastic kernel $Q\left(\cdot \mid a_{t}, x_{t+1}\right), t=0,1, \ldots$.

Define the observable histories: $h_{0}:=\left(p, y_{0}\right) \in \mathbb{H}_{0}$ and $h_{t}:=\left(p, y_{0}, a_{0}, \ldots, y_{t-1}, a_{t-1}, y_{t}\right) \in \mathbb{H}_{t}$ for all $t=1,2, \ldots$, where $\mathbb{H}_{0}:=\mathbb{P}(\mathbb{X}) \times \mathbb{Y}$ and $\mathbb{H}_{t}:=\mathbb{H}_{t-1} \times \mathbb{A} \times \mathbb{Y}$ if $t=1,2, \ldots$ A policy $\pi$ for the POMDP is defined as a sequence $\pi=\left\{\pi_{t}\right\}_{t=0,1, \ldots}$ of stochastic kernels $\pi_{t}$ on $\mathbb{A}$ given $\mathbb{H}_{t}$. A policy $\pi$ is called nonrandomized, if each probability measure $\pi_{t}\left(\cdot \mid h_{t}\right)$ is concentrated at one point. The set of all policies is denoted by $\Pi$. The Ionescu Tulcea theorem (Bertsekas and Shreve [5, pp. 140-141] or 
Hernández-Lerma and Lasserre [18, p.178]) implies that a policy $\pi \in \Pi$ and an initial distribution $p \in \mathbb{P}(\mathbb{X})$, together with the stochastic kernels $P, Q$ and $Q_{0}$, determine a unique probability measure $P_{p}^{\pi}$ on the set of all trajectories $(\mathbb{X} \times \mathbb{Y} \times \mathbb{A})^{\infty}$ endowed with the $\sigma$-field defined by the products of Borel $\sigma$-fields $\mathcal{B}(\mathbb{X})$, $\mathcal{B}(\mathbb{Y})$, and $\mathcal{B}(\mathbb{A})$. The expectation with respect to this probability measure is denoted by $\mathbb{E}_{p}^{\pi}$.

For a finite horizon $T=0,1, \ldots$, the expected total discounted costs are

$$
V_{T, \alpha}^{\pi}(p):=\mathbb{E}_{p}^{\pi} \sum_{t=0}^{T-1} \alpha^{t} c\left(x_{t}, a_{t}\right), \quad p \in \mathbb{P}(\mathbb{X}), \pi \in \Pi,
$$

where $\alpha \geq 0$ is the discount factor, $V_{0, \alpha}^{\pi}(p)=0$. Consider the following assumptions.

Assumption (D). $c$ is bounded below on $\mathbb{X} \times \mathbb{A}$ and $\alpha \in(0,1)$.

Assumption (P). $c$ is nonnegative on $\mathbb{X} \times \mathbb{A}$ and $\alpha=1$.

When $T=\infty$, formula (5.1) defines the infinite horizon expected total discounted cost, and we denote it by $V_{\alpha}^{\pi}(p)$. For any function $g^{\pi}(p)$, including $g^{\pi}(p)=V_{T, \alpha}^{\pi}(p)$ and $g^{\pi}(p)=V_{\alpha}^{\pi}(p)$, define the optimal values

$$
g(p):=\inf _{\pi \in \Pi} g^{\pi}(p), \quad p \in \mathbb{P}(\mathbb{X}) .
$$

A policy $\pi$ is called optimal for the respective criterion, if $g^{\pi}(p)=g(p)$ for all $p \in \mathbb{P}(\mathbb{X})$. For $g^{\pi}=V_{T, \alpha}^{\pi}$, the optimal policy is called T-horizon discount-optimal; for $g^{\pi}=V_{\alpha}^{\pi}$, it is called discount-optimal.

We recall that a function $c$ defined on $\mathbb{X} \times \mathbb{A}$ with values in $\overline{\mathbb{R}}^{1}$ is inf-compact if the set $\{(x, a) \in \mathbb{X} \times \mathbb{A}$ : $c(x, a) \leq \lambda\}$ is compact for any finite number $\lambda$. A function $c$ defined on $\mathbb{X} \times \mathbb{A}$ with values in $\overline{\mathbb{R}}^{1}$ is called $\mathbb{K}$-inf-compact on $\mathbb{X} \times \mathbb{A}$, if for any compact set $K \subseteq \mathbb{X}$, the function $c: K \times \mathbb{A} \rightarrow \overline{\mathbb{R}}^{1}$ defined on $K \times \mathbb{A}$ is inf-compact; Feinberg et al. [11, 13, Definition 1.1]. According to Feinberg et al. [13, Lemma 2.5], a bounded below function $c$ is $\mathbb{K}$-inf-compact on the product of metric spaces $\mathbb{X}$ and $\mathbb{A}$ if and only if it satisfies the following two conditions:

(a) $c$ is lower semi-continuous;

(b) if a sequence $\left\{x^{(n)}\right\}_{n=1,2, \ldots}$ with values in $\mathbb{X}$ converges and its limit $x$ belongs to $\mathbb{X}$ then any sequence $\left\{a^{(n)}\right\}_{n=1,2, \ldots}$ with $a^{(n)} \in \mathbb{A}, n=1,2, \ldots$, satisfying the condition that the sequence $\left\{c\left(x^{(n)}, a^{(n)}\right)\right\}_{n=1,2, \ldots}$ is bounded above, has a limit point $a \in \mathbb{A}$.

For a $\operatorname{POMDP}(\mathbb{X}, \mathbb{Y}, \mathbb{A}, P, Q, c)$, consider the $\operatorname{MDP}(\mathbb{X}, \mathbb{A}, P, c)$, in which all the states are observable. An MDP can be viewed as a particular POMDP with $\mathbb{Y}=\mathbb{X}$ and $Q(B \mid a, x)=Q(B \mid x)=\mathbf{I}\{x \in B\}$ for all $x \in \mathbb{X}, a \in \mathbb{A}$, and $B \in \mathcal{B}(\mathbb{X})$. In addition, for an MDP an initial state is observable. Thus for an MDP an initial state $x$ is considered instead of the initial distribution $p$. In fact, this MDP possesses a special property that action sets at all the states are equal.

It is well known that the analysis and optimization of an POMDP can be reduced to the analysis and optimization to a specially constructed MDPs called a COMDP. The states of the COMDP are posterior state distributions of the original POMDP. In order to find an optimal policy for POMDP, it is sufficient to find such a policy for the COMDP, and then it is easy to construct an optimal policy for the COMDPs (see Bertsekas and Shreve [5, Section 10.3], Dynkin and Yushkevich [10, Chapter 8], Hernández-Lerma [17, p. 87], Yushkevich [34] or Rhenius [24] for details). However, little is known about the existence of optimal policies for COMDPs and how to find them when the state, observation, and action sets are Borel spaces. 
The rest of this section presents recent results from Feinberg et al. [15] on the existence optimal policies and their computation for COMDPs and therefore for POMDPs.

Our next goal is to define the transition probability $q$ for the COMDP presented in (5.5). Given a posterior distribution $z$ of the state $x$ at time epoch $t=0,1, \ldots$ and given an action $a$ selected at epoch $t$, denote by $R(B \times C \mid z, a)$ the joint probability that the state at time $(t+1)$ belongs to the set $B \in \mathcal{B}(\mathbb{X})$ and the observation at time $t+1$ belongs to the set $C \in \mathcal{B}(\mathbb{Y})$,

$$
R(B \times C \mid z, a):=\int_{\mathbb{X}} \int_{B} Q\left(C \mid a, x^{\prime}\right) P\left(d x^{\prime} \mid x, a\right) z(d x), B \in \mathcal{B}(\mathbb{X}), C \in \mathcal{B}(\mathbb{Y}), z \in \mathbb{P}(\mathbb{X}), a \in \mathbb{A} .
$$

Observe that $R$ is a stochastic kernel on $\mathbb{X} \times \mathbb{Y}$ given $\mathbb{P}(\mathbb{X}) \times \mathbb{A}$; see Bertsekas and Shreve [5, Section 10.3], Dynkin and Yushkevich [10, Chapter 8], Hernández-Lerma [17, p. 87], Yushkevich [34], or Rhenius [24] for details. The probability that the observation $y$ at time $t+1$ belongs to the set $C \in \mathcal{B}(\mathbb{Y})$, given that at time $t$ the posterior state probability is $z$ and selected action is $a$, is $R^{\prime}(C \mid z, a):=R(\mathbb{X} \times C \mid z, a), C \in \mathcal{B}(\mathbb{Y})$, $z \in \mathbb{P}(\mathbb{X}), a \in \mathbb{A}$. Observe that $R^{\prime}$ is a stochastic kernel on $\mathbb{Y}$ given $\mathbb{P}(\mathbb{X}) \times \mathbb{A}$. By Bertsekas and Shreve [5], Proposition 7.27], there exist a stochastic kernel $H$ on $\mathbb{X}$ given $\mathbb{P}(\mathbb{X}) \times \mathbb{A} \times \mathbb{Y}$ such that

$$
R(B \times C \mid z, a)=\int_{C} H(B \mid z, a, y) R^{\prime}(d y \mid z, a), \quad B \in \mathcal{B}(\mathbb{X}), C \in \mathcal{B}(\mathbb{Y}), z \in \mathbb{P}(\mathbb{X}), a \in \mathbb{A} .
$$

The stochastic kernel $H(\cdot \mid z, a, y)$ defines a measurable mapping $H: \mathbb{P}(\mathbb{X}) \times \mathbb{A} \times \mathbb{Y} \rightarrow \mathbb{P}(\mathbb{X})$, where $H(z, a, y)(\cdot)=H(\cdot \mid z, a, y)$. For each pair $(z, a) \in \mathbb{P}(\mathbb{X}) \times \mathbb{A}$, the mapping $H(z, a, \cdot): \mathbb{Y} \rightarrow \mathbb{P}(\mathbb{X})$ is defined $R^{\prime}(\cdot \mid z, a)$-almost surely uniquely in $y \in \mathbb{Y}$; Bertsekas and Shreve [5, Corollary 7.27.1] or Dynkin and Yushkevich [10, Appendix 4.4]. For a posterior distribution $z_{t} \in \mathbb{P}(\mathbb{X})$, action $a_{t} \in \mathbb{A}$, and an observation $y_{t+1} \in \mathbb{Y}$, the posterior distribution $z_{t+1} \in \mathbb{P}(\mathbb{X})$ is

$$
z_{t+1}=H\left(z_{t}, a_{t}, y_{t+1}\right)
$$

However, the observation $y_{t+1}$ is not available in the COMDP model, and therefore $y_{t+1}$ is a random variable with the distribution $R^{\prime}\left(\cdot \mid z_{t}, a_{t}\right)$, and the right-hand side of (5.4) maps $\left(z_{t}, a_{t}\right) \in \mathbb{P}(\mathbb{X}) \times \mathbb{A}$ to $\mathbb{P}(\mathbb{P}(\mathbb{X}))$. Thus, $z_{t+1}$ is a random variable with values in $\mathbb{P}(\mathbb{X})$ whose distribution is defined uniquely by the stochastic kernel

$$
q(D \mid z, a):=\int_{\mathbb{Y}} \mathbf{I}\{H(z, a, y) \in D\} R^{\prime}(d y \mid z, a), \quad D \in \mathcal{B}(\mathbb{P}(\mathbb{X})), z \in \mathbb{P}(\mathbb{X}), a \in \mathbb{A} ;
$$

Hernández-Lerma [17, p. 87]. The particular choice of a stochastic kernel $H$ satisfying (5.3) does not effect the definition of $q$ from (5.5), since for each pair $(z, a) \in \mathbb{P}(\mathbb{X}) \times \mathbb{A}$, the mapping $H(z, a, \cdot): \mathbb{Y} \rightarrow \mathbb{P}(\mathbb{X})$ is defined $R^{\prime}(\cdot \mid z, a)$-almost surely uniquely in $y \in \mathbb{Y}$.

The COMDP is defined as an MDP with the parameters $(\mathbb{P}(\mathbb{X}), \mathbb{A}, q, \bar{c})$, where (i) $\mathbb{P}(\mathbb{X})$ is the state space; (ii) $\mathbb{A}$ is the action set available at all states $z \in \mathbb{P}(\mathbb{X})$; (iii) the one-step cost function $\bar{c}: \mathbb{P}(\mathbb{X}) \times \mathbb{A} \rightarrow \overline{\mathbb{R}}^{1}$, defined

$$
\bar{c}(z, a):=\int_{\mathbb{X}} c(x, a) z(d x), \quad z \in \mathbb{P}(\mathbb{X}), a \in \mathbb{A} ;
$$

(iv) transition probabilities $q$ on $\mathbb{P}(\mathbb{X})$ given $\mathbb{P}(\mathbb{X}) \times \mathbb{A}$ defined in (5.5). 
For an MDP, a nonrandomized policy is called Markov, if all decisions depend only on the current state and time. A Markov policy is called stationary, if all decisions depend only on current states.

For MDPs, Feinberg et al. [13, Theorem 2] provides general conditions for the existence of optimal policies, validity of optimality equations, and convergence of value iterations. Here we formulate these conditions for an MDP whose action sets in all states are equal, and then Theorem 5.1 adapts Feinberg et al. [13, Theorem 2] to POMDPs.

Assumption ( $\mathbf{W}^{*}$ ) (cf. Feinberg et al. [15] and Lemma 2.5 in [13]). (i) the function $c$ is $\mathbb{K}$-inf-compact on $\mathbb{X} \times \mathbb{A}$; (ii) the transition probability $P(\cdot \mid x, a)$ is weakly continuous in $(x, a) \in \mathbb{X} \times \mathbb{A}$.

For the COMDP, Assumption ( $\mathbf{W}^{*}$ ) has the following form: (i) $\bar{c}$ is $\mathbb{K}$-inf-compact on $\mathbb{P}(\mathbb{X}) \times \mathbb{A}$; (ii) the transition probability $q(\cdot \mid z, a)$ is weakly continuous in $(z, a) \in \mathbb{P}(\mathbb{X}) \times \mathbb{A}$.

In the following theorem, the notation $\bar{v}$ is used for the expected total costs for COMDPs instead the symbol $V$ used for POMDPs. The following theorem follows directly from Feinberg et al. [12, Theorem 2] applied to the COMDP $(\mathbb{P}(\mathbb{X}), \mathbb{A}, q, \bar{c})$.

Theorem 5.1. (Feinberg et al. [15, Theorem 3.1]). Let either Assumption (D) or Assumption (P) hold. If the COMDP $(\mathbb{P}(\mathbb{X}), \mathbb{A}, q, \bar{c})$ satisfies Assumption $\left(\mathbf{W}^{*}\right)$, then:

(i) the functions $\bar{v}_{t, \alpha}, t=0,1, \ldots$, and $\bar{v}_{\alpha}$ are lower semi-continuous on $\mathbb{P}(\mathbb{X})$, and $\bar{v}_{t, \alpha}(z) \rightarrow \bar{v}_{\alpha}(z)$ as $t \rightarrow \infty$ for all $z \in \mathbb{P}(\mathbb{X})$

(ii) for each $z \in \mathbb{P}(\mathbb{X})$ and $t=0,1, \ldots$,

$$
\begin{gathered}
\bar{v}_{t+1, \alpha}(z)=\min _{a \in \mathbb{A}}\left\{\bar{c}(z, a)+\alpha \int_{\mathbb{P}(\mathbb{X})} \bar{v}_{t, \alpha}\left(z^{\prime}\right) q\left(d z^{\prime} \mid z, a\right)\right\}= \\
\min _{a \in \mathbb{A}}\left\{\int_{\mathbb{X}} c(x, a) z(d x)+\alpha \int_{\mathbb{X}} \int_{\mathbb{X}} \int_{\mathbb{Y}} \bar{v}_{t, \alpha}(H(z, a, y)) Q\left(d y \mid a, x^{\prime}\right) P\left(d x^{\prime} \mid x, a\right) z(d x)\right\},
\end{gathered}
$$

where $\bar{v}_{0, \alpha}(z)=0$ for all $z \in \mathbb{P}(\mathbb{X})$, and the nonempty sets

$$
A_{t, \alpha}(z):=\left\{a \in \mathbb{A}: \bar{v}_{t+1, \alpha}(z)=\bar{c}(z, a)+\alpha \int_{\mathbb{P}(\mathbb{X})} \bar{v}_{t, \alpha}\left(z^{\prime}\right) q\left(d z^{\prime} \mid z, a\right)\right\}, \quad z \in \mathbb{P}(\mathbb{X}), t=0,1, \ldots
$$

satisfy the following properties: (a) the graph $\operatorname{Gr}\left(A_{t, \alpha}\right)=\left\{(z, a): z \in \mathbb{P}(\mathbb{X}), a \in A_{t, \alpha}(z)\right\}, t=0,1, \ldots$, is a Borel subset of $\mathbb{P}(\mathbb{X}) \times \mathbb{A}$, and $(b)$ if $\bar{v}_{t+1, \alpha}(z)=+\infty$, then $A_{t, \alpha}(z)=\mathbb{A}$ and, if $\bar{v}_{t+1, \alpha}(z)<+\infty$, then $A_{t, \alpha}(z)$ is compact;

(iii) for each $T=1,2, \ldots$, for the COMDP there exists an optimal Markov $T$-horizon policy $\left(\phi_{0}, \ldots, \phi_{T-1}\right)$, and if for a T-horizon Markov policy $\left(\phi_{0}, \ldots, \phi_{T-1}\right)$ the inclusions $\phi_{T-1-t}(z) \in A_{t, \alpha}(z), z \in \mathbb{P}(\mathbb{X})$, $t=0, \ldots, T-1$, hold, then this policy is T-horizon optimal;

(iv) for each $z \in \mathbb{P}(\mathbb{X})$

$$
\begin{gathered}
\bar{v}_{\alpha}(z)=\min _{a \in \mathbb{A}}\left\{\bar{c}(z, a)+\alpha \int_{\mathbb{P}(\mathbb{X})} \bar{v}_{\alpha}\left(z^{\prime}\right) q\left(d z^{\prime} \mid z, a\right)\right\}= \\
\min _{a \in \mathbb{A}}\left\{\int_{\mathbb{X}} c(x, a) z(d x)+\alpha \int_{\mathbb{X}} \int_{\mathbb{X}} \int_{\mathbb{Y}} \bar{v}_{\alpha}(H(z, a, y)) Q\left(d y \mid a, x^{\prime}\right) P\left(d x^{\prime} \mid x, a\right) z(d x)\right\},
\end{gathered}
$$


and the nonempty sets

$$
A_{\alpha}(z):=\left\{a \in \mathbb{A}: \bar{v}_{\alpha}(z)=\bar{c}(z, a)+\alpha \int_{\mathbb{P}(\mathbb{X})} \bar{v}_{\alpha}\left(z^{\prime}\right) q\left(d z^{\prime} \mid z, a\right)\right\}, \quad z \in \mathbb{P}(\mathbb{X}),
$$

satisfy the following properties: (a) the graph $\operatorname{Gr}\left(A_{\alpha}\right)=\left\{(z, a): z \in \mathbb{P}(\mathbb{X}), a \in \mathbb{A}_{\alpha}(z)\right\}$ is a Borel subset of $\mathbb{P}(\mathbb{X}) \times \mathbb{A}$, and $(b)$ if $\bar{v}_{\alpha}(z)=+\infty$, then $A_{\alpha}(z)=\mathbb{A}$ and, if $\bar{v}_{\alpha}(z)<+\infty$, then $A_{\alpha}(z)$ is compact.

(v) for an infinite horizon problem there exists a stationary discount-optimal policy $\phi_{\alpha}$ for the COMDP, and a stationary policy $\phi_{\alpha}^{*}$ for the COMDP is optimal if and only if $\phi_{\alpha}^{*}(z) \in A_{\alpha}(z)$ for all $z \in \mathbb{P}(\mathbb{X})$.

(vi) if $\bar{c}$ is inf-compact on $\mathbb{P}(\mathbb{X}) \times \mathbb{A}$, then the functions $\bar{v}_{t, \alpha}, t=1,2, \ldots$, and $\bar{v}_{\alpha}$ are inf-compact on $\mathbb{P}(\mathbb{X})$.

Theorem 5.1 establishes the existence of stationary optimal policies, validity of optimality equations, and convergence of value iterations to optimal values under the following natural conditions: (i) Assumption (D) or (P) and the function $\bar{c}$ is $K$-inf-compact, and (ii) the stochastic kernel $q$ on $\mathbb{P}(\mathbb{X})$ given $\mathbb{P}(\mathbb{X}) \times A$ is weakly continuous. Theorems 5.2 and 5.3 provide sufficient conditions for (i) and (ii) respectively in terms of the properties of the cost function $c$ and stochastic kernels $P$ and $Q$.

Theorem 5.2. (Feinberg et al. [15, Theorem 3.4]). If the stochastic kernel $P\left(d x^{\prime} \mid x, a\right)$ on $\mathbb{X}$ given $\mathbb{X} \times \mathbb{A}$ is weakly continuous and the cost function $c: \mathbb{X} \times \mathbb{A} \rightarrow \overline{\mathbb{R}}^{1}$ is bounded below and $\mathbb{K}$-inf-compact on $\mathbb{X} \times \mathbb{A}$, then the cost function $\bar{c}: \mathbb{P}(\mathbb{X}) \times \mathbb{A} \rightarrow \overline{\mathbb{R}}^{1}$ defined for the COMDP in (5.6) is bounded from below by the same constant as $c$ and $\mathbb{K}$-inf-compact on $\mathbb{P}(\mathbb{X}) \times \mathbb{A}$.

Theorem 5.3. (Feinberg et al. [15, Theorem 3.7]). The weak continuity of the stochastic kernel $P\left(d x^{\prime} \mid x, a\right)$ on $\mathbb{X}$ given $\mathbb{X} \times \mathbb{A}$ and continuity in the total variation of the stochastic kernel $Q(d y \mid a, x)$ on $\mathbb{Y}$ given $\mathbb{A} \times \mathbb{X}$ imply that the stochastic kernel $q\left(d z^{\prime} \mid z, a\right)$ on $\mathbb{P}(\mathbb{X})$ given $\mathbb{P}(\mathbb{X}) \times \mathbb{A}$ is weakly continuous.

The following assumption, that has similarities with (4.3), and theorem are used in Feinberg et al. [15] to prove Theorem 5.3 .

Assumption (H). There exists a stochastic kernel $H$ on $\mathbb{X}$ given $\mathbb{P}(\mathbb{X}) \times \mathbb{A} \times \mathbb{Y}$ satisfying (5.3) such that: if a sequence $\left\{z^{(n)}\right\}_{n=1,2, \ldots} \subseteq \mathbb{P}(\mathbb{X})$ converges weakly to $z \in \mathbb{P}(\mathbb{X})$, and a sequence $\left\{a^{(n)}\right\}_{n=1,2, \ldots} \subseteq \mathbb{A}$ converges to $a \in \mathbb{A}$ as $n \rightarrow \infty$, then there exists a subsequence $\left\{\left(z^{\left(n_{k}\right)}, a^{\left(n_{k}\right)}\right)\right\}_{k=1,2, \ldots} \subseteq\left\{\left(z^{(n)}, a^{(n)}\right)\right\}_{n=1,2, \ldots}$ and a measurable subset $C$ of $\mathbb{Y}$ such that $R^{\prime}(C \mid z, a)=1$ and for all $y \in C$

$$
H\left(z^{\left(n_{k}\right)}, a^{\left(n_{k}\right)}, y\right) \text { converges weakly to } H(z, a, y) \text {. }
$$

In other words, 5.9) holds $R^{\prime}(\cdot \mid z, a)$-almost surely.

According to the following theorem, if the stochastic kernel $R^{\prime}$ is setwise continuous and Assumption $(\mathbf{H})$ holds, then the stochastic kernel $q$ is weakly continuous. According to Feinberg et al. [15, Theorem 3.7], weak continuity of the stochastic kernel $P$ and continuity of the observation stochastic kernel $Q$ in the total variation imply that the stochastic kernel $R^{\prime}$ is setwise continuous and Assumption (H) holds. Another sufficient condition for weak continuity of $q$ is that there is a weakly continuous version of a stochastic kernel $H$ on $\mathbb{X}$ given $\mathbb{P}(\mathbb{X}) \times \mathbb{A} \times \mathbb{Y}$; see Striebel [33] and Hernández-Lerma [17]. However, this condition may not hold for a POMDP with a weakly continuous stochastic kernel $P$ and a observation stochastic kernel $Q$ continuous in the total observation; see Feinberg et al. [15, Example 4.2]. 
Theorem 5.4. (Feinberg et al. [15, Theorem 3.5]). If the stochastic kernel $R^{\prime}(d y \mid z, a)$ on $\mathbb{Y}$ given $\mathbb{P}(\mathbb{X}) \times$ $\mathbb{A}$ is setwise continuous and Assumption $(\mathbf{H})$ holds, then the stochastic kernel $q\left(d z^{\prime} \mid z, a\right)$ on $\mathbb{P}(\mathbb{X})$ given $\mathbb{P}(\mathbb{X}) \times \mathbb{A}$ is weakly continuous.

In addition to Theorem 5.3, that provides the sufficient condition of weak continuity of a stochastic kernel $q$ in terms of transition and observation probabilities $P$ and $Q$, and to Theorem 5.4 that provides the sufficient condition of weak continuity of a stochastic kernel $q$ in terms of stochastic kernels $R^{\prime}$ and $H$, a sufficient condition can be formulated in terms of the stochastic kernel $R$ on $\mathbb{X} \times \mathbb{Y}$ given $\mathbb{P}(\mathbb{X}) \times \mathbb{A}$, defined in (5.2). For each $B \in \tau(\mathbb{X})$ consider the family of functions

$$
\mathcal{R}_{B}=\{\mathbb{P}(\mathbb{X}) \times \mathbb{A} \rightarrow R(B \times C \mid z, a): C \in \tau(\mathbb{Y})\}
$$

mapping $\mathbb{P}(\mathbb{X}) \times \mathbb{A}$ into $[0,1]$.

Theorem 5.5. Let the topology on $\mathbb{X}$ have a countable base $\tau_{b}^{\mathbb{X}}$ with the following two properties:

(a) $\mathbb{X} \in \tau_{b}^{\mathbb{X}}$

(b) for each finite intersection $\mathcal{O}=\cap_{i=1}^{k} \mathcal{O}_{i}$ of sets $\mathcal{O}_{i} \in \tau_{b}^{\mathbb{X}}, i=1,2, \ldots, k$, the family of functions $\mathcal{R}_{\mathcal{O}}$ is equicontinuous at all the points $(z, a) \in \mathbb{P}(\mathbb{X}) \times \mathbb{A}$.

Then the following two statements take place:

(i) the stochastic kernel $R^{\prime}(d y \mid z, a)$ on $\mathbb{Y}$ given $\mathbb{P}(\mathbb{X}) \times \mathbb{A}$ is continuous in the total variation, and Assumption (H) holds;

(ii) the stochastic kernel $q\left(d z^{\prime} \mid z, a\right)$ on $\mathbb{P}(\mathbb{X})$ given $\mathbb{P}(\mathbb{X}) \times \mathbb{A}$ is weakly continuous.

Proof. (i) The equicontinuity at all the points $(z, a) \in \mathbb{P}(\mathbb{X}) \times \mathbb{A}$ of the family of functions $\mathcal{R}_{\mathcal{O}}$ defined on $\mathbb{P}(\mathbb{X}) \times \mathbb{A}$, being applied to $\mathcal{O}=\mathbb{X}$, implies that the stochastic kernel $R^{\prime}$ on $\mathbb{X}$ given $\mathbb{P}(\mathbb{X}) \times \mathbb{A}$ is continuous in the total variation. Theorem 4.4 being applied to the Borel subsets of Polish spaces $\mathbb{S}_{1}=\mathbb{X}, \mathbb{S}_{2}=\mathbb{Y}$, and $\mathbb{S}_{3}=\mathbb{P}(\mathbb{X}) \times \mathbb{A}$, yields that Assumption (H) holds. (ii) Since the continuity of $R^{\prime}$ in the total variations implies its setwise continuity, the statement follows from statement (i) and Theorem 5.4

The following theorem completes the descriptions of the relations between the assumptions of Theorems 5.3 5.5 Among these three groups of assumptions, the assumptions of Theorem 5.4 are the most general, and they follow from the assumptions of Theorem 5.5, which in its turn follow from the assumptions of Theorem 5.3 .

Theorem 5.6. If the stochastic kernel $P\left(d x^{\prime} \mid x, a\right)$ on $\mathbb{X}$ given $\mathbb{X} \times \mathbb{A}$ is weakly continuous and the stochastic kernel $Q(d y \mid a, x)$ on $\mathbb{Y}$ given $\mathbb{A} \times \mathbb{X}$ is continuous in the total variation, then the assumptions of Theorem 5.5 hold.

Proof. In view of Feinberg et al. [15, Lemma 5.3], the family of function $\mathcal{R}_{\mathcal{O}_{1} \backslash \mathcal{O}_{2}}$ is equicontinuous for two arbitrary open subsets $\mathcal{O}_{1}$ and $\mathcal{O}_{2}$ in $\mathbb{X}$. By setting $\mathcal{O}_{2}=\emptyset$, this result implies that the family of functions $\mathcal{R}_{\mathcal{O}}$ is equicontinuous for each open subset $\mathcal{O}$ in $\mathbb{X}$. Since we endowed $\mathbb{X}$ with the induced topology from a separable metric space, its topology has a countable base which is closed according to the finite intersections. Therefore, this countable base of the topology on $\mathbb{X}$ satisfies assumptions of Theorem 5.5 . 
Observe that Theorem 5.3 follows from Theorems 5.5 and 5.6 The following theorem provides sufficient conditions for the existence of optimal policies for the COMDP. Its first statement is Theorem 5.3, which is repeated for completeness of the statements.

Theorem 5.7. (Feinberg et al. [15, Theorem 3.6]). Let either Assumption (D) or Assumption (P) hold. If the function $c$ is $\mathbb{K}$-inf-compact on $\mathbb{X} \times \mathbb{A}$ then each of the following conditions:

(i) the stochastic kernel $P\left(d x^{\prime} \mid x, a\right)$ on $\mathbb{X}$ given $\mathbb{X} \times \mathbb{A}$ is weakly continuous, and the stochastic kernel $Q(d y \mid a, x)$ on $\mathbb{Y}$ given $\mathbb{A} \times \mathbb{X}$ is continuous in the total variation;

(ii) the assumptions of Theorem 5.5 hold;

(iii) the stochastic kernel $R^{\prime}(d y \mid z, a)$ on $\mathbb{Y}$ given $\mathbb{P}(\mathbb{X}) \times \mathbb{A}$ is setwise continuous and Assumption $(\mathbf{H})$ holds,

implies that the COMDP $(\mathbb{P}(\mathbb{X}), \mathbb{A}, q, \bar{c})$ satisfies Assumption $\left(\mathbf{W}^{*}\right)$, and therefore statements $(i)-(v i)$ of Theorem 5.1 hold.

Proof. Theorem 5.2 implies that the cost function $\bar{c}$ for the COMDP is bounded below and $\mathbb{K}$-inf-compact on $\mathbb{P}(\mathbb{X}) \times \mathbb{A}$. Weak continuity of the stochastic kernel $q$ on $\mathbb{P}(\mathbb{X})$ given $\mathbb{P}(\mathbb{X}) \times \mathbb{A}$ follows from Theorems 5.3 5.5

Example 4.1 from Feinberg et al. [15] demonstrates that, if the stochastic kernel $Q(d y \mid a, x)$ on $\mathbb{Y}$ given $\mathbb{A} \times \mathbb{X}$ is setwise continuous, then the transition probability $q$ for the COMDP may not be weakly continuous in $(z, a) \in \mathbb{P}(\mathbb{X}) \times \mathbb{A}$. In that example the state set consists of two points. Therefore, if the stochastic kernel $P\left(d x^{\prime} \mid x, a\right)$ on $\mathbb{X}$ given $\mathbb{X} \times \mathbb{A}$ is setwise continuous (even if it is continuous in the total variation) in $(x, a) \in \mathbb{X} \times \mathbb{A}$ then the setwise continuity of the stochastic kernel $Q(d y \mid a, x)$ on $\mathbb{Y}$ given $\mathbb{A} \times \mathbb{X}$ is not sufficient for the weak continuity of $q$.

\section{Markov Decision Models with Incomplete Information}

Consider a Markov decision model with incomplete information (MDMII); Dynkin and Yushkevich [10, Chapter 8], Rhenius [24], Yushkevich [34] (see also Rieder [25] and Bäuerle and Rieder [3] for a version of this model with transition probabilities having densities). This model is defined by an observed state space $\mathbb{Y}$, an unobserved state space $\mathbb{W}$, an action space $\mathbb{A}$, nonempty sets of available actions $A(y)$, where $y \in \mathbb{Y}$, a stochastic kernel $P$ on $\mathbb{Y} \times \mathbb{W}$ given $\mathbb{Y} \times \mathbb{W} \times \mathbb{A}$, and a one-step cost function $c: G \rightarrow \overline{\mathbb{R}}^{1}$, where $G=\{(y, w, a) \in \mathbb{Y} \times \mathbb{W} \times \mathbb{A}: a \in A(y)\}$ is the graph of the mapping $A(y, w)=A(y),(y, w) \in \mathbb{Y} \times \mathbb{W}$. Assume that:

(i) $\mathbb{Y}, \mathbb{W}$ and $\mathbb{A}$ are Borel subsets of Polish spaces. For all $y \in \mathbb{Y}$ a nonempty Borel subset $A(y)$ of $\mathbb{A}$ represents the set of actions available at $y$;

(ii) the graph of the mapping $A: \mathbb{Y} \rightarrow 2^{\mathbb{A}}$, defined as $\operatorname{Gr}(A)=\{(y, a): y \in \mathbb{Y}, a \in A(y)\}$ is measurable, that is, $\operatorname{Gr}(A) \in \mathcal{B}(\mathbb{Y} \times \mathbb{A})$, and this graph allows a measurable selection, that is, there exists a measurable mapping $\phi: \mathbb{Y} \rightarrow \mathbb{A}$ such that $\phi(y) \in A(y)$ for all $y \in \mathbb{Y}$; 
(iii) the stochastic kernel $P$ on $\mathbb{X}$ given $\mathbb{Y} \times \mathbb{W} \times \mathbb{A}$ is weakly continuous in $(y, w, a) \in \mathbb{Y} \times \mathbb{W} \times \mathbb{A}$;

(iv) the one-step cost function $c$ is $\mathbb{K}$-inf-compact on $G$, that is, for each compact set $K \subseteq \mathbb{Y} \times \mathbb{W}$ and for each $\lambda \in \mathbb{R}^{1}$, the set $\mathcal{D}_{K, c}(\lambda)=\{(y, w, a) \in G: c(y, w, a) \leq \lambda\}$ is compact.

Let us define $\mathbb{X}=\mathbb{Y} \times \mathbb{W}$, and for $x=(y, w) \in \mathbb{X}$ let us define $Q(C \mid x)=\mathbf{I}\{y \in C\}$ for all $C \in \mathcal{B}(\mathbb{Y})$. Observe that this $Q$ corresponds to the continuous function $y=F(x)$, where $F(y, w)=y$ for all $x=(y, w) \in \mathbb{X}$ (here $F$ is a projection of $\mathbb{X}=\mathbb{Y} \times \mathbb{W}$ on $\mathbb{Y}$ ). Thus, as explained in Example 4.1 from Feinberg et al. [15], the stochastic kernel $Q(d y \mid x)$ is weakly continuous in $x \in \mathbb{X}$. Then by definition, an MDMII is a POMDP with the state space $\mathbb{X}$, observation set $\mathbb{Y}$, action space $\mathbb{A}$, available action sets $A(y)$, stochastic kernel $P$, observation kernel $Q(d y \mid a, x):=Q(d y \mid x)$, and one-step cost function $c$. However, this model differs from our basic definition of a POMDP because action sets $A(y)$ depend on observations and one-step costs $c(x, a)=c(y, w, a)$ are not defined when $a \notin A(y)$. To avoid this difficulty, we set $c(y, w, a)=+\infty$ when $a \notin A(y)$. The extended function $c$ is $\mathbb{K}$-inf-compact on $\mathbb{X} \times \mathbb{A}$ because the set $\mathcal{D}_{K, c}(\lambda)$ remains unchanged for each $K \subseteq \mathbb{Y} \times \mathbb{W}$ and for each $\lambda \in \mathbb{R}^{1}$.

Thus, an MDMII is a special case of a $\operatorname{POMDP}(\mathbb{X}, \mathbb{Y}, \mathbb{A}, P, Q, c)$, when $\mathbb{X}=\mathbb{Y} \times \mathbb{W}$ and the observation kernel $Q$ is defined by the projection of $\mathbb{X}$ on $\mathbb{Y}$. The observation stochastic kernel $Q(\cdot \mid x)$ is weakly continuous in $x \in \mathbb{X}$. This is weaker that the continuity of $Q$ in the total variation that, according to Theorem 5.7 ensures weak continuity of the stochastic kernel for the COMDP and the existence of optimal policie. Indeed, Feinberg et al. [15, Example 8.1] demonstrates that even under the stronger assumption, that $P$ is setwise continuous, the corresponding stochastic kernel $q$ on $\mathbb{P}(\mathbb{X})$ given $\mathbb{P}(X) \times \mathbb{A}$ may not be weakly continuous.

The natural question is: which conditions are sufficient for the existence of optimal policies for the MDMII? Since an MDMII is a particular POMDP, the existence of optimal policies for an MDMII is equivalent to the existence of optimal policies for the COMDP corresponding to this MDMII. Theorem 5.1 gives an answer in a general form by stating that such conditions are the week continuity of the transition probability $q$ of the corresponding COMDP and the $\mathbb{K}$-inf-compactness of the cost function $\bar{c}$ for the COMDP. The following theorem provides a sufficient condition for the weak continuity of $q$. For each open set $\mathcal{O}$ in $\mathbb{W}$ consider the family of functions $\mathcal{P}_{\mathcal{O}}^{*}=\{(x, a) \rightarrow P(C \times \mathcal{O} \mid x, a): C \in \tau(\mathbb{Y})\}$ mapping $\mathbb{X} \times \mathbb{A}$ into $[0,1]$.

Theorem 6.1. Let the topology on $\mathbb{W}$ have a countable base $\tau_{b}^{\mathbb{W}}$ satisfying the following two conditions:

(i) $\mathbb{W} \in \tau_{b}^{\mathbb{W}}$

(ii) for each finite intersection $\mathcal{O}=\cap_{i=1}^{k} \mathcal{O}_{i}$ of sets $\mathcal{O}_{i} \in \tau_{b}^{\mathbb{W}}, i=1,2, \ldots, k$, the family of functions $\mathcal{P}_{\mathcal{O}}^{*}$ is equicontinuous at all the points $(x, a) \in \mathbb{X} \times \mathbb{A}$.

Then the stochastic kernel $q\left(d z^{\prime} \mid z, a\right)$ on $\mathbb{P}(\mathbb{X})$ given $\mathbb{P}(\mathbb{X}) \times \mathbb{A}$ is weakly continuous.

Proof. Let $\tau_{b}^{\mathbb{Y}}$ be a countable base of the topology on $\mathbb{Y}$ closed with respect to the finite intersections. Such base exists, because $\mathbb{Y}$ is the separable metric space. Since finite intersections of elements of the base $\tau_{b}^{\mathbb{W}}$ are open sets, let us choose $\tau_{b}^{\mathbb{W}}$ in a way that finite intersections of elements of $\tau_{b}^{\mathbb{W}}$ belong to $\tau_{b}^{\mathbb{W}}$. Then $\tau_{b}^{\mathbb{X}}:=\left\{\mathcal{O}_{\mathbb{Y}} \times \mathcal{O}_{\mathbb{W}}: \mathcal{O}_{\mathbb{Y}} \in \tau_{b}^{\mathbb{Y}}, \mathcal{O}_{\mathbb{W}} \in \tau_{b}^{\mathbb{W}}\right\}$ is the countable base of the topology on $\mathbb{X}=\mathbb{Y} \times \mathbb{W}$ defined by the products of the topologies on $\mathbb{Y}$ and $\mathbb{W}$ and for any finite tuples of open sets $\left\{\mathcal{O}_{\mathbb{Y}}^{(j)}\right\}_{j=1}^{N}$ in $\mathbb{Y}$ and 
$\left\{\mathcal{O}_{\mathbb{W}}^{(j)}\right\}_{j=1}^{N}$ in $\mathbb{W}, N=1,2, \ldots$, their finite intersections $\cap_{j=1}^{N} \mathcal{O}_{\mathbb{Y}}^{(j)}$ and $\cap_{j=1}^{N} \mathcal{O}_{\mathbb{W}}^{(j)}$ are open in $\mathbb{Y}$ and $\mathbb{W}$ respectively. Moreover, $\cap_{j=1}^{N}\left(\mathcal{O}_{\mathbb{Y}}^{(j)} \times \mathcal{O}_{\mathbb{W}}^{(j)}\right)=\left(\cap_{j=1}^{N} \mathcal{O}_{\mathbb{Y}}^{(j)}\right) \times\left(\cap_{j=1}^{N} \mathcal{O}_{\mathbb{W}}^{(j)}\right) \in \tau_{b}^{\mathbb{X}}$ for any finite tuples of open sets $\left\{\mathcal{O}_{\mathbb{Y}}^{(j)}\right\}_{j=1}^{N}$ from $\tau_{b}^{\mathbb{Y}}$ and $\left\{\mathcal{O}_{\mathbb{W}}^{(j)}\right\}_{j=1}^{N}$ from $\tau_{b}^{\mathbb{W}}$. From (5.2) it follows that

$$
\begin{gathered}
R\left(C_{1} \times B \times C_{2} \mid z, a\right)=\int_{\mathbb{X}} P\left(\left(C_{1} \cap C_{2}\right) \times B \mid x, a\right) z(d x), \quad B \in \mathcal{B}(\mathbb{W}), C_{1}, C_{2} \in \mathcal{B}(\mathbb{Y}), z \in \mathbb{P}(\mathbb{X}), a \in \mathbb{A}, \\
R^{\prime}(C \mid z, a)=\int_{\mathbb{X}} P(C \times \mathbb{W} \mid x, a) z(d x), \quad C \in \mathcal{B}(\mathbb{Y}), z \in \mathbb{P}(\mathbb{X}), a \in \mathbb{A} .
\end{gathered}
$$

For any nonempty open sets $\mathcal{O}_{\mathbb{Y}} \in \tau_{b}^{\mathbb{Y}}$ and $\mathcal{O}_{\mathbb{W}} \in \tau_{b}^{\mathbb{W}}$ respectively, Theorem 4.2, with $\mathbb{S}_{1}=\mathbb{P}(\mathbb{X}), \mathbb{S}_{2}=\mathbb{X}$, $\mathbb{S}_{3}=\mathbb{A}, \mathcal{O}=\mathbb{X}, \Psi(B \mid z)=z(B)$, and $\left.\mathcal{A}_{0}=\left\{(x, a) \rightarrow P\left(\left(\mathcal{O}_{\mathbb{Y}} \cap C\right) \times \mathcal{O}_{\mathbb{W}}\right) \mid x, a\right): C \in \tau(\mathbb{Y})\right\}$, implies the equicontinuity of the family of functions

$$
\mathcal{R}_{\mathcal{O}_{\mathbb{Y}} \times \mathcal{O}_{\mathbb{W}}}=\left\{(z, a) \rightarrow R\left(\mathcal{O}_{\mathbb{Y}} \times \mathcal{O}_{\mathbb{W}} \times C \mid z, a\right): C \in \tau(\mathbb{Y})\right\}
$$

defined on $\mathbb{P}(\mathbb{X}) \times \mathbb{A}$, at all the points $(z, a) \in \mathbb{P}(\mathbb{X}) \times \mathbb{A}$. Therefore, Theorem 5.5 (ii) yields that the stochastic kernel $q\left(d z^{\prime} \mid z, a\right)$ on $\mathbb{P}(\mathbb{X})$ given $\mathbb{P}(\mathbb{X}) \times \mathbb{A}$ is weakly continuous.

Assumptions of Theorem 6.1 are weaker than equicontinuity at all the points $(x, a) \in \mathbb{X} \times \mathbb{A}$ of the family of functions $\mathcal{P}_{\mathcal{O}}$ for all open sets $\mathcal{O}$ in $\mathbb{W}$ (see Example 4.10 above), which in its turn is a weaker assumption than the continuity of the stochastic kernel $P$ on $\mathbb{X}$ given $\mathbb{X} \times \mathbb{A}$ in the total variation. The following theorem states sufficient conditions for the existence of optimal policies for MDMIIs, the validity of optimality equations, and convergence of value iterations to optimal values. Theorem 6.2 generalizes [15, Theorem 8.2], where the equicontinuity at all the points $(x, a) \in \mathbb{X} \times \mathbb{A}$ of the family of functions $\mathcal{P}_{\mathcal{O}}^{*}$ for all open sets $\mathcal{O}$ in $\mathbb{W}$ is assumed.

Theorem 6.2. Let either Assumption (D) or Assumption (P) hold, and let the cost function $c$ be $\mathbb{K}$-infcompact on $G$. If the topology on $\mathbb{W}$ has a countable base $\tau_{b}^{\mathbb{W}}$ satisfying assumptions (i) and (ii) of Theorem 6.1 then the COMDP $(\mathbb{P}(\mathbb{X}), \mathbb{A}, q, \bar{c})$ satisfies Assumption $\left(\mathbf{W}^{*}\right)$, and therefore the conclusions of Theorem 5.1 hold.

Proof. Assumption $\left(\mathbf{W}^{*}\right)(\mathrm{i})$ follows from Corollary 4.1 and Theorem 5.2, Assumption ( $\left.\mathbf{W}^{*}\right)$ (ii) follows from Theorem 6.1 Therefore, the $\operatorname{COMDP}(\mathbb{P}(\mathbb{X}), \mathbb{A}, q, \bar{c})$ satisfies Assumption $\left(\mathbf{W}^{*}\right)$ and the conclusions of Theorem 5.1 hold.

Acknowledgements. The authors thank M. Mandava for providing useful remarks. The research of the first author was partially supported by NSF grant CMMI-1335296.

\section{References}

[1] Aoki, M. (1965) Optimal control of partially observable Markovian systems. J. Franklin Inst. 280(5): 367-386.

[2] Ash R.B. (1972) Real Analysis and Probability, Academic Press, New York. 
[3] Bäuerle N, Rieder U (2011) Markov Decision Processes with Applications to Finance, Springer-Verlag, Berlin.

[4] Bensoussan A (1992) Stochastic Control of Partially Observable Systems, Cambridge University Press, Cambridge, UK.

[5] Bertsekas D.P., Shreve S.E. (1978) Stochastic Optimal Control: The Discrete-Time Case, Academic Press, New York; reprinted by Athena Scientific, Belmont, MA, 1996.

[6] Billingsley, P. (1968) Convergence of Probability Measures, Jonh Wiley, New York.

[7] Bogachev V.I. (2007) Measure Theory, Volume II, Springer-Verlag, Berlin.

[8] Cohn D.L. (2013) Measure Theory (Springer, New York).

[9] Dynkin, E.B. (1965) Controlled random sequences. Theory Probab. Appl. 10(1): 1-14.

[10] Dynkin, E.B., Yushkevich A.A. (1979) Controlled Markov Processes, Springer-Verlag, New York.

[11] Feinberg E.A., Kasyanov P.O., Voorneveld M. (2014) Berge's maximum theorem for noncompact image sets, J. Math. Anal. Appl. 413(2): 1040-1046.

[12] Feinberg E.A., Kasyanov P.O., Zadoianchuk N.V. (2012) Average-cost Markov decision processes with weakly continuous transition probabilities. Math. Oper. Res. 37(4): 591-607.

[13] Feinberg E.A., Kasyanov P.O., Zadoianchuk N.V. (2013) Berge's theorem for noncompact image sets, J. Math. Anal. Appl. 397(1): 255-259.

[14] Feinberg E.A., Kasyanov P.O., Zgurovsky M.Z. (2013) Optimality conditions for total-cost partially observable Markov decision processes, Proceedings of the 52th IEEE Conference on Decision and Control and European Control Conference, Florence, Italy, December 10-13, 2013, 5716-5721.

[15] Feinberg E.A., Kasyanov P.O., Zgurovsky M.Z. (2014) Partially observable total-cost Markov decision processes with weakly continuous transition probabilities. arXiv:1401.2168.

[16] Feinberg E.A., Kasyanov P.O., Zgurovsky M.Z. (2014) Optimality conditions for partially observable Markov decision processes, in Continuous and Distributed Systems. Theory and Applications (eds. M.Z. Zgurovsky, V.A. Sadovnichy), Springer, New York, 251-264.

[17] Hernández-Lerma O. (1989) Adaptive Markov Control Processes, Springer-Verlag, New York.

[18] Hernández-Lerma O., Lassere J.B. (1996) Discrete-Time Markov Control Processes: Basic Optimality Criteria, Springer, New York.

[19] Hinderer K. (1970) Foundations of Non-stationary Dynamic Programming with Discrete Time Parameter, Springer-Verlag, Berlin. 
[20] Jacod J., Shiryaev A.N. (2003) Limit Theorems for Stochastic Processes, Second edition, Springer, Berlin.

[21] Kabanov Yu.M., Liptser R. Sh., Shiryaev, A.N. (1980) Some limit theorems for simple point processes (a martingale approach). Stochastics 3: 203-216.

[22] Liptser R. Sh., Shiryaev, A.N. (1974) Statistics of Random Processes. Nonlinear Filtering and Related Problems. Nauka, Moscow (in Russiand). Engl. transl.: Statistics of Random Processes. I. General Theory. II. Applications. Springer-Verlag, New York, 1977, 1978.

[23] Parthasarathy K.R. (1967) Probability Measures on Metric Spaces, Academic Press, New York.

[24] Rhenius D. (1974) Incomplete information in Markovian decision models. Ann. Statist. 2(6): 13271334.

[25] Rieder, U. (1975) Bayesian dynamiv programming. Adv. Appl. Probab. 7(2): 330-348.

[26] Royden H.L. (1968) Real Analysis, Second edition, Macmillan, New York.

[27] Sawaragi Y., Yoshikawa T. (1970) Descrete-time markovian decision processes with incomplete state observations. Ann. Math. Statist. 41(1): 78-86.

[28] Shiryaev A.N. (1964) On the theory of decision functions and control by observation from incomplete data. Transactions of the Third Prague Conference on Information Theory, Statistical Decision Functions, Random Processes (Liblice, 1962), pp. 657-681 (in Russian); Engl. transl. in Select. Transl. Math. Statist. Probab. 6(1966), 162-188.

[29] Shiryaev A.N. (1967) Some new results in the theory of controlled random processes. Transactions of the Fourth Prague Conference on Information Theory, Statistical Decision Functions, Random Processes (Prague, 1965), pp. 131-201 (in Russian); Engl. transl. in Select. Transl. Math. Statist. Probab. 8(1969), 49-130.

[30] Shiryaev A.N. (1996) Probability, Second edition, Springer-Verlag, New York.

[31] Smallwood RD, Sondik EJ (1973) The optimal control of partially observable Markov processes over a finite horizon. Oper. Res. 21(5): 1071-1088.

[32] Sondik EJ (1978) The optimal control of partially observable Markov processes over the infinite horizon: Discounted costs. Oper. Res, 26(2): 282-304.

[33] Striebel C (1975) Optimal Control for Discrete Time Stochastic Systems, Springer-Verlag, Berlin.

[34] Yushkevich A.A. (1976) Reduction of a controlled Markov model with incomplete data to a problem with complete information in the case of Borel state and control spaces. Theory Probab. Appl. 21(1): 153-158. 